\title{
Optimal design of a structured fixed-bed reactor using geometry optimization and Stratoconception printing process
}

\author{
Alexis Courtais $^{1}$, François Lesage ${ }^{1}$, Yannick Privat ${ }^{2}$, Cyril Pelaingre ${ }^{3}$, and \\ Abderrazak M. Latifi ${ }^{1, *}$ \\ ${ }^{1}$ Laboratoire Réactions et Génie des Procédés, CNRS-ENSIC, Université de Lorraine, 1 rue de \\ Grandville, BP20451, 54001, Nancy Cedex, France \\ ${ }^{2}$ Institut de Recherche Mathématique Avancée, CNRS, Université de Strasbourg, 7 rue \\ René-Descartes, 67084, Strasbourg Cedex, France \\ ${ }^{3}$ Centre Européen de Prototypage et Outillage Rapide, CIRTES, 29 bis voie de l'innovation, \\ 88100 Saint-Dié-des-Vosges, France \\ *abderrazak.latifi@univ-lorraine.fr
}

\begin{abstract}
The aim of this paper is to determine the shape of a fixed-bed reactor which maximizes the conversion rate under the constraints of process model equations (i.e. continuity, Navier-Stokes, and mass balance equations), energy dissipation, iso-volume, and manufacturing. Incompressible fluid, laminar flow regime and steady-state conditions in the reactor are the main assumptions taken into account. The optimization method developed is based on the adjoint system method and OpenFOAM framework is used as CFD solver to compute the state vector and its adjoint variables introduced by the optimization approach. The algorithm developed is then tested on two different cases, a reactor where a first order homogeneous reaction takes place and another one involving a surface reaction. The optimization results show a significant improvement of the conversion rate by $2.7 \%$ in the first case, and by $16 \%$ in the second one. Finally, initial and optimal shapes are manufactured using a $3 \mathrm{D}$ printing technique.
\end{abstract}

Keywords: Shape optimization, Adjoint system method, Computational fluid dynamics, OpenFOAM environment, Fixed-bed reactor, Additive manufacturing

\section{Introdution}

The objective of shape optimization is to deform the outer boundary of an object in order to minimize or maximize a performances index, while satisfying given constraints. Historically, shape optimization methods have been used in cutting-edge technologies mainly in advanced areas such as aerodynamics (Burgreen et al., 1994; Reuther et al., 1999; Hicks and Henne, 1978). However, they have recently been extended to other engineering areas where the shape greatly influences the performances. For example, in hydrodynamics, the 

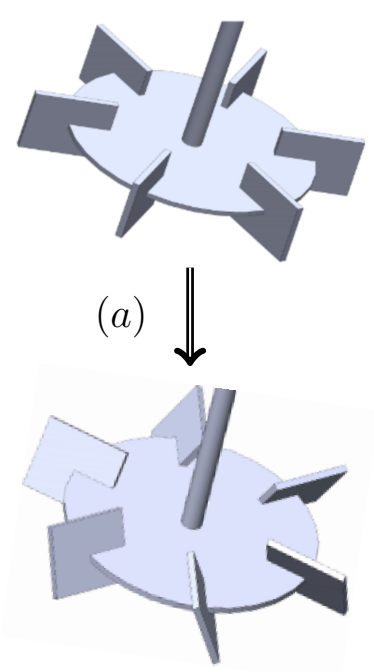

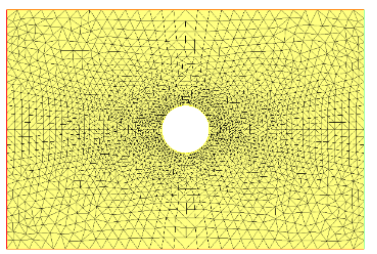

(b)

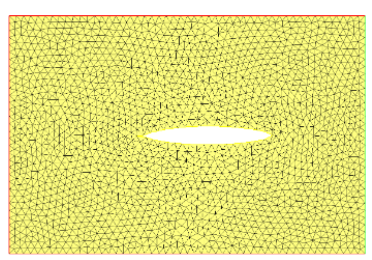

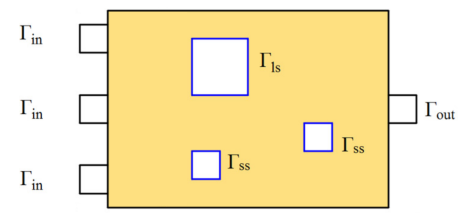

(c)

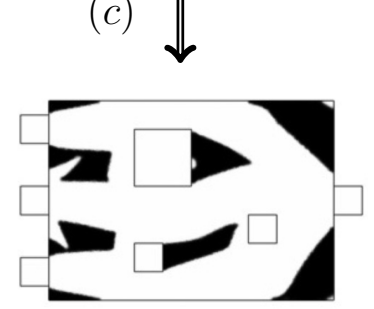

Figure 1: Example of optimized shapes using (a) parameter optimization - optimization of the angle and the thickness of an agitator blades (Hoseini et al., 2020), (b) geometry optimization - determination of the obstacle shape that minimizes the pressure drops (Dapogny et al., 2018) and (c) topology optimization - determination of the optimized topology and shape of a heat exchanger (Dong and Liu, 2020).

shape of a pipe that minimizes the energy dissipated by the fluid due to viscous friction was analyzed (Tonomura et al., 2010; Henrot and Privat, 2010; Courtais et al., 2019).

In chemical engineering however, where the shape of unit operations (e.g. reactors, tanks, stirrers, pipes...) is an important design parameter, the shape optimization has not been extensively investigated. This important issue deserves therefore to be addressed and will probably result in a paradigm shift in optimal design and operation of processes.

Shape optimization methods can be grouped into 3 main families illustrated in Fig. 1:

(a) The first family is parameter optimization (Lin et al., 2011; Kundu, 2007) where the general shape of the object to design is known and the optimization method can only modify some parameters chosen by the user. Figure 1(a) presents the shape optimization of an agitator where only the thickness and the angle of the blades are the decision variables.

(b) The second one is geometry optimization where the decision variables are no longer defined by some parameters but by the boundary of the optimized object (Courtais et al., 2019; Henrot and Privat, 2010). This kind of family allows a deformation of the global shape, but, it prevents topology changes. In 2D, it means that the number of holes or inclusions remains invariable during the optimization process. Figure 1(b) presents the determination of the obstacle shape that minimizes the pressure drops. Since a geometry algorithm is used, the obstacle cannot be removed.

(c) The last one is topology optimization which is an extension of geometry optimization 
and allows topology changes (Dong and Liu, 2020; Zhou et al., 2018). In Fig. 1(c), the two-dimensional topology optimization of a heat exchanger is shown. In this case, the topology is one of the decision variables.

On the one hand, parameter optimization methods are by far the most developed in chemical engineering area since they are the most straightforward to implement, computationally less expensive than the other methods, and the determined shapes are easily manufacturable since the general shape is chosen by the user. However, they do not allow significant modifications of the shape, and consequently lead to poor optimization performances. Indeed, parameter optimization methods consist in reformulating the shape optimization problem as a static optimization problem, and therefore provide limited flexibility since the number of decision variables is low. For example, Hoseini et al. (2020) optimized the shape of an agitator through three geometrical parameters of the blades such as the thickness and the vertical angle (Fig. 1), Liang and Yuan (2020) modified the configuration of a Y-shape microreactor through the position and the width of a sudden contraction of the microreactor section, and Grundtvig et al. (2017) optimized the configuration of a bio-catalytic microreactor by adapting the position of 140 boundary points and then interpolating to build the outer boundary of the reactor.

On the other hand, topology optimization approaches such as the level-set (Kambampati et al., 2021) or the homogenization (Ozguc et al., 2021) methods are mainly developed for optimal design of structures in mechanical engineering. They allow to explore all possible shapes and topologies during the optimization process, and therefore lead to interesting performance improvements. However, those methods are more CPU time consuming and the optimal geometry could be complex and not straightforward to manufacture (Allaire et al., 2017).

Geometry optimization methods offer an adequate compromise between the advantages and drawbacks of topology and parameter approaches. Indeed, they allow the analysis of a wide range of shapes while preserving the manufacturability of the final shape even if it can be complex in practice. For this purpose, the recent development of additive manufacturing techniques can provide a 3D printing solution since they allow to extend the set of realistic shapes but require to consider some additional constraints. Two of these constraints impose a minimum thickness on the printed solid part and a minimum distance between solid elements. A possible methodology for their treatments can be found in Allaire et al. (2016), however, in this paper, we provide a simpler alternative well adapted to the application considered.

The present work is devoted to the development of a geometry optimization algorithm using the adjoint system method. The resulting algorithm is used in optimal design of fixed-bed reactors where

(i) a first order homogeneous reaction takes place,

(ii) a surface reaction limited by the external mass transfer occurs.

These two cases will thereafter be referred to as (HR) and (SR), respectively. In both cases, the objective is to find the shape of the packing which minimizes the average concentration of the reactant at the outlet of the reactor, i.e. maximizes the conversion 
rate of the reactor, while meeting the constraints of (i) process model, (ii) iso-volume maintaining the same hydraulic residence time between initial and optimal reactors, (iii) energy dissipation, and (iv) manufacturing. The resulting optimal shapes will then be tested numerically by means of residence time distribution computations.

The paper is organized as follows. First, the optimization problems are defined (Section 2) and their mathematical formulations are presented (Section 3). Section 4 describes the implementation of the algorithm within OpenFOAM framework. Finally, Section 5 is devoted to the presentation of the numerical results and the manufactured optimal shapes.

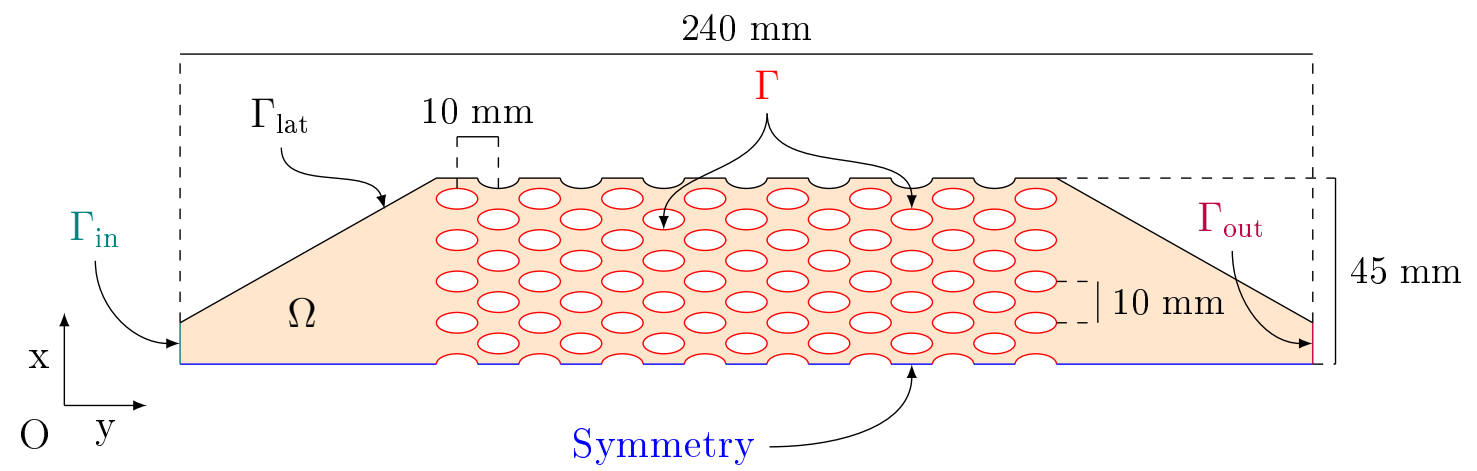

Figure 2: Initial shape of the reactor used for the optimization process $\Omega_{0}$. A symmetry axis is located in the reactor center on which symmetry boundary conditions are imposed.

\section{Presentation of the optimization problems considered}

\subsection{Case studies and their modeling}

In this work, the process considered is a fixed-bed reactor where a single-phase liquid flows. The initial structure of the packing consists of elliptical obstacles (whose half axes are 5 $\mathrm{mm}$ and $2.5 \mathrm{~mm}$ ) uniformly distributed in the reactor. Fig. 2 illustrates schematically the initial configuration of the reactor to be optimized as well as its dimensions.

The reactor is denoted $\Omega$ (see Fig. 2) and is delimited by the union of the fluid inlet $\left(\Gamma_{\text {in }}\right)$, outlet $\left(\Gamma_{\text {out }}\right)$, lateral wall $\left(\Gamma_{\text {lat }}\right)$ and free $(\Gamma)$ boundaries. This last boundary represents the reactor packing and stands for the decision variable of the optimization problem. It is the only boundary that will evolve during the optimization process.

The fluid flow in the reactor $\Omega$ is modeled by the momentum balance described by the Navier-Stokes and the continuity equations and the associated boundary conditions: (a) no slip condition applied on walls $\Gamma_{\text {lat }}$ and $\Gamma$, (b) a flow velocity imposed at the inlet, and (c) the normal stress tensor component imposed equal to zero at the outlet. The system 
of equations is therefore expressed as:

$$
\left\{\begin{aligned}
-\nu \Delta \boldsymbol{U}+(\boldsymbol{U} \cdot \nabla \boldsymbol{U})+\nabla p=0 & \text { in } \Omega \\
\nabla \cdot \boldsymbol{U}=0 & \text { in } \Omega \\
\boldsymbol{U}=\boldsymbol{U}_{\text {in }} & \text { on } \Gamma_{\text {in }} \\
\boldsymbol{U}=0 & \text { on } \Gamma_{\text {lat }} \cup \Gamma \\
\sigma(\boldsymbol{U}, p) \boldsymbol{n}=0 & \text { on } \Gamma_{\text {out }}
\end{aligned}\right.
$$

where $\boldsymbol{n}$ is the boundary normal vector, $\nu$ and $p$ are the kinematic viscosity and $p$ the kinematic pressure (i.e. the absolute pressure divided by the fluid density), respectively. According to equations (1a) and (1b), the fluid flow is assumed stationary, incompressible, and the relative pressure is imposed equal to zero at the reactor outlet. $\sigma(\boldsymbol{U}, p)$ is the stress tensor divided by the fluid density. It is defined by

$$
\sigma(\boldsymbol{U}, p)=2 \nu \varepsilon(\boldsymbol{U})-p \boldsymbol{I} \text { with } \varepsilon(\boldsymbol{U})=\frac{1}{2}\left(\nabla \boldsymbol{U}+(\nabla \boldsymbol{U})^{T}\right)
$$

where $\boldsymbol{I}$ is the identity matrix.

At the inlet boundary $\Gamma_{\text {in }}$, the velocity profile is uniform and follows (Oy) direction. It is set to a small value in order to impose low particle Reynolds number (see Eq. (3)) ensuring a laminar flow in the reactor.

$$
\operatorname{Re}_{\mathrm{p}}=\frac{u d_{H}}{\nu}
$$

where $u$ is the superficial velocity and $d_{H}=\frac{4 S}{P}$ is the hydraulic diameter with $S=\pi a b$ the surface of the elliptical obstacles ( $a$ and $b$ are the half axes) and $P \approx \pi \sqrt{2\left(a^{2}+b^{2}\right)}$ their perimeter. In the initial configuration, the particle Reynolds number does not exceed 7 which justifies the laminar flow assumption. It will be verified in the optimal shapes of the reactor.

The mass transfer modelling is presented for the two cases (HR) and (SR) in the following subsections.

\subsubsection{First case study: homogeneous reaction}

The first reaction considered is of type $\mathrm{R} \rightarrow \mathrm{P}$, homogeneous and of first order with respect to the reactant. Its kinetics is expressed as:

$$
r=k C
$$

where $k$ is the kinetic constant and $C$ the concentration of the reactant $\mathrm{R}$. In this case, we assume that the reaction only takes place in the fluid phase (i.e. in $\Omega$ ), the packing just acts as a static mixer. Thus, the mass balance on $\mathrm{R}$ is described by the following system of PDEs:

$$
\left\{\begin{aligned}
-\mathcal{D} \Delta C+\boldsymbol{U} \cdot \nabla C+k C=0 & \text { in } \Omega \\
C=C_{\text {in }} & \text { on } \Gamma_{\text {in }} \\
\frac{\partial C}{\partial \boldsymbol{n}}=0 & \text { on } \Gamma_{\text {lat }} \cup \Gamma_{\text {out }} \cup \Gamma
\end{aligned}\right.
$$


where $\mathcal{D}$ is the mass diffusion coefficient of the reactant $\mathrm{R}$ in the solvant.

\subsubsection{Second case study: surface reaction}

The second reaction is heterogeneous, limited by the external mass transfer and occurs on walls $\Gamma$ and $\Gamma_{\text {lat }}$. It is assumed that the packing $\Gamma$ and the lateral wall $\Gamma_{\text {lat }}$ are both catalyst impregnated and the reaction is very fast at the surface of catalyst leading to null concentration on those boundaries. All these assumptions lead to the following mass balance equations:

$$
\left\{\begin{aligned}
-\mathcal{D} \Delta C+\boldsymbol{U} \cdot \nabla C=0 & \text { in } \Omega \\
C=C_{\text {in }} & \text { on } \Gamma_{\text {in }} \\
C=0 & \text { on } \Gamma_{\text {lat }} \cup \Gamma \\
\frac{\partial C}{\partial \boldsymbol{n}}=0 & \text { on } \Gamma_{\text {out }}
\end{aligned}\right.
$$

\subsection{Shape optimization problems}

The shape optimization problems are defined by:

- a performance index to be minimized. It is defined by the average concentration of the reactant at the reactor outlet. Such a performance index is relevant since the conversion rate depends on the average concentration of the reactant at the outlet. It is given by:

$$
J(\Omega)=\int_{\Gamma_{\text {out }}} C d \sigma
$$

where $C$ is the solution of (5) or (6) depending on the case.

- decision variables. In a shape optimization problem the decision variable is the shape of the domain described by the free boundary $\Gamma$.

- a process model. It is described by the Navier-Stokes momentum equations without turbulence model, the continuity Eqs. (1) and the mass balance equations (5) or (6).

- a set of constraints. Here, four constraints are considered. The first constraint is an iso-volume constraint defined in order to maintain the same residence time between initial and optimal shapes. The second constraint is an inequality constraint on energy dissipation by the fluid due to viscous friction. Such a constraint is relevant since the energy dissipation is proportional to the pressure drop. The two constraints are given by the following relations:

$$
\begin{gathered}
C_{\mathcal{V}}(\Omega)=\mathcal{V}(\Omega)-\mathcal{V}\left(\Omega_{0}\right)=0 \\
C_{\mathcal{E}}(\Omega)=2 \nu \int_{\Omega}|\varepsilon(\boldsymbol{U})|^{2} d x-\underbrace{2 \nu \int_{\Omega_{0}}|\varepsilon(\boldsymbol{U})|^{2} d x}_{\mathcal{E}_{0}} \leqslant 0
\end{gathered}
$$


with $|\varepsilon(\boldsymbol{U})|^{2}=\varepsilon(\boldsymbol{U}): \varepsilon(\boldsymbol{U})$. The notation ":" is the double inner product of two tensors defined by $A: B=\sum_{i, j=1}^{3} A_{i, j} B_{i, j}$. In Eqs. (8) and (9), $\mathcal{V}(\Omega)$ represents the volume of $\Omega$ and $(\boldsymbol{U}, p)$ is the solution of Eqs. (1).

The two last constraints take into account the manufacturability of the optimal shape of the object to be designed. These constraints involve a minimum distance between two obstacles and a minimum thickness of obstacles. Since the differentiation with respect to the domain is a complex task (Feppon et al., 2020), these constraints are not included in the Lagrangian, their treatment is detailed in paragraph 4.1.

The Lagrangian of the problem which aggregates the performance index, the volume and energy constraints is defined as:

$$
\mathcal{L}\left(\Omega, \lambda_{\mathcal{V}}, \lambda_{\mathcal{E}}\right)=K_{\text {crit }} J(\Omega)+\lambda_{\mathcal{V}} C_{\mathcal{V}}(\Omega)+\lambda_{\mathcal{E}} C_{\mathcal{E}}(\Omega)
$$

where $K_{\text {crit }}$ is a constant ensuring dimensional consistency of the terms of the Lagrangian functional, $\lambda_{\mathcal{V}}$ and $\lambda_{\mathcal{E}}$ are the Lagrange multipliers respectively associated to volume and energy constraints.

In conclusion, the shape optimization problem is formulated as:

$$
\begin{array}{ll}
\min _{\Omega} & J(\Omega) \\
\text { s.t. } & \Omega \in \mathcal{C} \\
& (\boldsymbol{U}, p) \text { solution of Eqs. (1) } \\
& C \text { solution of Eqs. (5) or (6) }
\end{array}
$$

where $\mathcal{C}$ represents the set of constraints given by

$$
\mathcal{C}:=\left\{\Omega \subset \mathbb{R}^{2} \mid C_{\mathcal{V}}(\Omega)=0 \text { and } C_{\mathcal{E}}(\Omega) \leqslant 0\right\} .
$$

\section{Shape optimization method: adjoint system method}

The method developed to solve the formulated shape optimization problem is based on geometry optimization. It is an iterative method which computes the gradient of the performance index and the constraints by means of the adjoint system method. Since two systems of PDEs (i.e. systems of momentum and mass balance equations) are involved in the model of each case studied, two adjoint systems are therfore introduced.

\subsection{Fundamental principle of the Hadamard method}

In the field of geometry optimization, the shape of an object is optimized by varying its boundaries which can be classified into two categories:

- fixed boundaries which will not be distorted during the optimization process. In this study, the boundaries $\Gamma_{\text {in }}, \Gamma_{\text {out }}$ and $\Gamma_{\text {lat }}$ are fixed.

- free boundaries which are the decision variables of the problem. In our cases, the free boundary is $\Gamma$. 
It is interesting to consider the largest possible free boundary in order to increase the degree of freedom of the optimization method. This will allow to reach a wider range of possible shapes, which will give better optimization performances. However, it will increase the number of local minima. The classification of the boundaries is the choice of the engineer who wants to optimize the object and depends mainly on the process involved (position of the fluid inlet and outlet, external boundary of the object, etc.).

The method used in this work is an iterative method that determines, from an initial shape of an object, a sequence of shapes that improve the performances of the object at each iteration by adapting the position of its boundaries. It is based on Hadamard's approach (Hadamard, 1908) and relies on the concept of derivative with respect to the domain, also called derivative in the sense of Hadamard (Allaire and Schoenauer, 2007; Henrot and Pierre, 2005). It consists in determining at each iteration the sensitivity of the performance index or the Lagrangian with respect to a small perturbation of the boundaries $\partial \Omega$ according to the following relation:

$$
\Omega_{i+1}=(\operatorname{Id}+t \boldsymbol{V})\left(\Omega_{i}\right)
$$

where Id is the identity operator, $t$ is the method step of the iterative algorithm, $i$ is the iteration index and $\boldsymbol{V}$ is the vector field standing for the perturbation. Fig. 3 illustrates the displacement of $\Omega$ during an iteration.

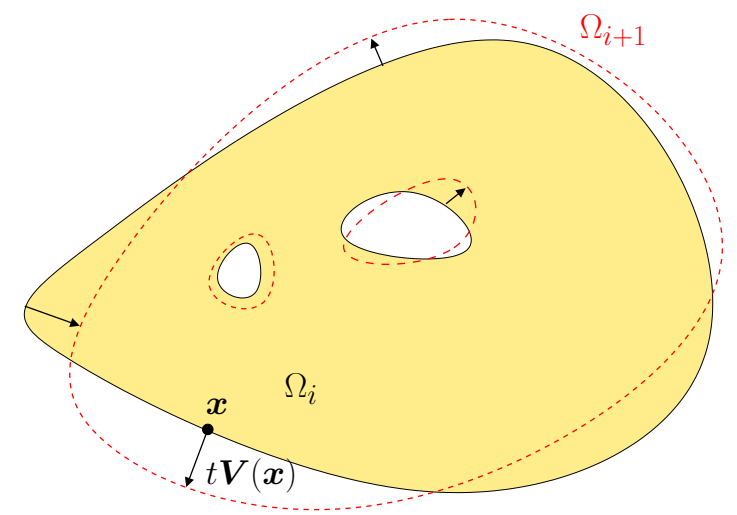

Figure 3: Example of an iteration of the shape optimization method. $t \boldsymbol{V}(\boldsymbol{x})$ is the small perturbation at point $\boldsymbol{x} \in \partial \Omega, \Omega_{i}$ represents the domain at iteration $i$.

The approach is based on the recurrence formula (13) and the objective of the method is to determine, at each iteration, the step $t$ and the vector field $\boldsymbol{V}$ leading to a decrease of the Lagrangian.

The derivative in the sense of Hadamard is a concept of direction derivative. The Lagrangian derivative following the direction $\boldsymbol{V}$ is computed with the following formula (Henrot and Pierre, 2005):

$$
\mathcal{L}^{\prime}\left(\Omega, \lambda_{\mathcal{V}}, \lambda_{\mathcal{E}}\right)(\boldsymbol{V})=\lim _{t \rightarrow 0} \frac{\mathcal{L}\left(\Omega_{t}, \lambda_{\mathcal{V}}, \lambda_{\mathcal{E}}\right)-\mathcal{L}\left(\Omega, \lambda_{\mathcal{V}}, \lambda_{\mathcal{E}}\right)}{t}
$$


with $\Omega_{t}=(\operatorname{Id}+t \boldsymbol{V})(\Omega)$.

\subsection{Adjoint system method}

The derivative in the sense of Hadamard can be decomposed in the same way as standard derivatives. Thus, the differential with respect to the domain of a sum is equal to the sum of the differentials:

$$
\mathcal{L}^{\prime}\left(\Omega, \lambda_{\mathcal{V}}, \lambda_{\mathcal{E}}\right)(\boldsymbol{V})=K_{\text {crit }} J^{\prime}(\Omega)(\boldsymbol{V})+\lambda_{\mathcal{V}} C_{\mathcal{V}}^{\prime}(\Omega)(\boldsymbol{V})+\lambda_{\mathcal{E}} C_{\mathcal{E}}^{\prime}(\Omega)(\boldsymbol{V})
$$

Standard differentiation formulae with respect to the domain applied to each term (Allaire and Schoenauer, 2007; Henrot and Pierre, 2005; Dapogny et al., 2018) yield

$$
\begin{gathered}
J^{\prime}(\Omega)(\boldsymbol{V})=\left.\frac{d}{d t} \int_{\Gamma_{\text {out }}} C d x\right|_{t=0}=\int_{\Gamma_{\text {out }}} C^{\prime} d \sigma \\
C_{\mathcal{V}}^{\prime}(\Omega)(\boldsymbol{V})=\frac{d}{d t}\left(\left.\int_{\Omega_{t}} 1 d x\right|_{t=0}-\mathcal{V}\left(\Omega_{0}\right)\right)=\int_{\partial \Omega}(\boldsymbol{V} \cdot \boldsymbol{n}) d \sigma \\
C_{\mathcal{E}}^{\prime}(\Omega)(\boldsymbol{V})=\frac{d}{d t}\left(\left.2 \nu \int_{\Omega_{t}}|\varepsilon(\boldsymbol{U})|^{2} d x\right|_{t=0}-\mathcal{E}\left(\Omega_{0}\right)\right) \\
=2 \nu \int_{\partial \Omega}|\varepsilon(\boldsymbol{U})|^{2}(\boldsymbol{V} \cdot \boldsymbol{n}) d \sigma+4 \nu \int_{\Omega} \varepsilon(\boldsymbol{U}): \varepsilon\left(\boldsymbol{U}^{\prime}\right) d x
\end{gathered}
$$

In Eqs. (16), (17) and (18), $\boldsymbol{U}^{\prime}$ describes the sensitivity of $\boldsymbol{U}$ with respect to the variation of $\Omega$. According to De La Sablonière et al. (2011) and Henrot and Privat (2010), $U^{\prime}$ is the solution of the following system of equations:

$$
\left\{\begin{aligned}
-\nu \Delta \boldsymbol{U}^{\prime}+\left(\boldsymbol{U}^{\prime} \cdot \nabla\right) \boldsymbol{U}+(\boldsymbol{U} \cdot \nabla) \boldsymbol{U}^{\prime}+\nabla p^{\prime} & =0 \\
\nabla \cdot \boldsymbol{U}^{\prime}=0 & \text { in } \Omega \\
\boldsymbol{U}^{\prime}=0 & \text { on } \Gamma_{\text {in }} \cup \Gamma_{\text {lat }} \\
\boldsymbol{U}^{\prime}=-\frac{\partial \boldsymbol{U}}{\partial \boldsymbol{n}}(\boldsymbol{V} \cdot \boldsymbol{n}) & \text { on } \Gamma \\
\sigma\left(\boldsymbol{U}^{\prime}, p^{\prime}\right) \boldsymbol{n}=0 & \text { on } \Gamma_{\text {out }}
\end{aligned}\right.
$$

where $\frac{\partial \boldsymbol{U}}{\partial n}=\nabla \boldsymbol{U} \boldsymbol{n}$ is the partial derivative with respect to the normal $\boldsymbol{n}$.

Similarly, $C^{\prime}$ represents the sensitivity of the concentration $C$ with respect to the variation of the domain. For the (HR) case, $C^{\prime}$ is the solution of the following system of PDEs (Courtais et al., 2021).

$$
\left\{\begin{aligned}
-\mathcal{D} \Delta C^{\prime}+\boldsymbol{U} \cdot \nabla C^{\prime}+\boldsymbol{U}^{\prime} \cdot \nabla C+k C^{\prime} & =0 \text { in } \Omega \\
C^{\prime} & =0 \text { on } \Gamma_{\text {in }} \\
\frac{\partial C^{\prime}}{\partial \boldsymbol{n}}=\mathcal{K}(C, \boldsymbol{V}) & \text { on } \Gamma \\
\frac{\partial C^{\prime}}{\partial \boldsymbol{n}} & =0 \text { on } \Gamma_{\text {out }} \cup \Gamma_{\text {lat }}
\end{aligned}\right.
$$


with $\mathcal{K}(C, \boldsymbol{V})=-\frac{\partial C^{2}}{\partial n^{2}}(\boldsymbol{V} \cdot \boldsymbol{n})+\nabla C \cdot(\nabla(\boldsymbol{V} \cdot \boldsymbol{n})-(\nabla(\boldsymbol{V} \cdot \boldsymbol{n}) \cdot \boldsymbol{n}) \boldsymbol{n})$. In the above system, equation (20b) comes from the usual differentiation formulae, equations (20a) and (20d) from the differentiation formula of a product and from a clever adaptation of Schwarz theorem (Henrot and Pierre, 2005, Chapter 5), and equation (20c) is a formula of differentiation with respect to the domain for a Neumann-type boundary condition on the free boundary (Henrot and Pierre, 2005, Chapter 5). For the case SR, $C^{\prime}$ is the solution of the following system of PDEs (Courtais, 2021):

$$
\left\{\begin{array}{rlrl}
-\mathcal{D} \Delta C^{\prime}+\boldsymbol{U} \cdot \nabla C^{\prime}+\boldsymbol{U}^{\prime} \cdot \nabla C & =0 & & \text { in } \Omega \\
C^{\prime} & =0 & & \text { on } \Gamma_{\text {in }} \cup \Gamma_{\text {lat }} \\
C^{\prime}=-\frac{\partial C}{\partial \boldsymbol{n}}(\boldsymbol{V} \cdot \boldsymbol{n}) & & \text { on } \Gamma \\
\frac{\partial C^{\prime}}{\partial \boldsymbol{n}} & =0 & & \text { on } \Gamma_{\text {out }}
\end{array}\right.
$$

Following Eqs. (16), (17) and (18), the derivative of the Lagrangian rewrites as follows:

$$
\begin{aligned}
\mathcal{L}^{\prime}\left(\Omega, \lambda_{\mathcal{V}}, \lambda_{\mathcal{E}}\right)(\boldsymbol{V})= & K_{\text {crit }} \int_{\Gamma_{\text {out }}} C^{\prime} d \sigma+\lambda_{\mathcal{V}} \int_{\partial \Omega}(\boldsymbol{V} \cdot \boldsymbol{n}) d \sigma \\
& +2 \nu \lambda_{\mathcal{E}}\left(\int_{\partial \Omega}|\varepsilon(\boldsymbol{U})|^{2}(\boldsymbol{V} \cdot \boldsymbol{n}) d \sigma+2 \int_{\Omega} \varepsilon(\boldsymbol{U}): \varepsilon\left(\boldsymbol{U}^{\prime}\right) d x\right)
\end{aligned}
$$

The above expression is not very usable for practical purposes. Indeed, some terms of equation (22) do not depend explicitly on the scalar product $(\boldsymbol{V} \cdot \boldsymbol{n})$. The dependence is achieved through $\boldsymbol{U}^{\prime}$. Under this form, it is complex to choose an appropriate perturbation $\boldsymbol{V}$ leading to a decrease of the Lagrangian functional. It is therefore more suitable to express the Lagrangian derivative in the following form:

$$
\mathcal{L}^{\prime}\left(\Omega, \lambda_{\mathcal{V}}, \lambda_{\mathcal{E}}\right)(\boldsymbol{V})=\int_{\partial \Omega} G\left(\Omega, \lambda_{\mathcal{V}}, \lambda_{\mathcal{E}}\right)(\boldsymbol{V} \cdot \boldsymbol{n})
$$

where $G\left(\Omega, \lambda_{\mathcal{V}}, \lambda_{\mathcal{E}}\right)$ is the shape gradient, a function defined on the boundary of the domain $\partial \Omega$ that depends on the solution of the Navier-Stokes equations $(\boldsymbol{U}, p)$ and the solution of the mass balance system $C$. To compute the gradient, adjoint system method is used, based on the introduction of two adjoint states: one associated to the Navier-Stokes equations $\left(\boldsymbol{U}_{\boldsymbol{a}}, p_{a}\right)$ and the other associated to the mass balance system $C_{a}$. Finally, from the values of the function $G\left(\Omega, \lambda_{\mathcal{V}}, \lambda_{\mathcal{E}}\right)$, the mesh displacement leading to a decrease of the Lagrangian is computed solving the following system (Courtais, 2021):

$$
\left\{\begin{aligned}
-\gamma \Delta \boldsymbol{V}+\boldsymbol{V}=0 & \text { in } \Omega \\
\boldsymbol{V}=0 & \text { on } \Gamma_{\text {in }} \cup \Gamma_{\text {out }} \cup \Gamma_{\text {lat }} \\
\gamma \nabla \boldsymbol{V} \boldsymbol{n}=-G\left(\Omega, \lambda_{\mathcal{V}}, \lambda_{\mathcal{E}}\right) \boldsymbol{n} & \text { on } \Gamma
\end{aligned}\right.
$$

where $\gamma$ is a positive parameter allowing to diffuse more or less the mesh displacement. This parameter must be properly chosen. Indeed, if its value is too low, the diffusion of 
the mesh will be small and the resulting free boundary surface will not be smooth. On the other hand, if the value of $\gamma$ is chosen too large, the displacement of the whole domain will mainly depend on high shape gradient areas.

Once the vector field $\boldsymbol{V}$ determined, the mesh is moved according to the discretized recurrence relation (13) expressed as follows:

$$
\Omega_{i+1}=(\boldsymbol{X}+t \boldsymbol{V})\left(\Omega_{i}\right)
$$

where $\boldsymbol{X}$ is the vector field of mesh points coordinates at iteration $i$.

Finally, all that remains is to determine the shape gradient $G\left(\Omega, \lambda_{\mathcal{V}}, \lambda_{\mathcal{E}}\right)$. Since each case involves a particular model, the expression of the shape gradient and the introduced adjoint states are presented separately.

\subsection{First case: homogeneous reaction}

Courtais et al. (2021) have detailed all calculations allowing to express the shape gradient in the following form:

$$
G\left(\Omega, \lambda_{\mathcal{V}}, \lambda_{\mathcal{E}}\right)=2 \nu\left(\varepsilon(\boldsymbol{U}): \varepsilon\left(\boldsymbol{U}_{\boldsymbol{a}}\right)-\lambda_{\mathcal{E}} \varepsilon(\boldsymbol{U}): \varepsilon(\boldsymbol{U})\right)-\frac{K_{\text {crit }}}{K_{\mathrm{BC}}} \mathcal{D} C_{a} \Delta C+\lambda_{\mathcal{V}}
$$

where $K_{\mathrm{BC}}$ is a constant present in the concentration outlet of the adjoint boundary conditions (28d) and (30c) allowing to homogenize Eqs. (26), (28d) and (30c). In Eq. (26), $\left(\boldsymbol{U}_{\boldsymbol{a}}, p_{a}\right)$ is the adjoint state of $(\boldsymbol{U}, p)$ defined as the solution of the following system:

$$
\left\{\begin{aligned}
\mathcal{H}\left(\boldsymbol{U}, \boldsymbol{U}_{a}\right)+\nabla p_{a}=-\frac{K_{\mathrm{crit}}}{K_{\mathrm{BC}}} C_{a} \nabla C & \text { in } \Omega \\
\nabla \cdot \boldsymbol{U}_{\boldsymbol{a}}=0 & \text { in } \Omega \\
\boldsymbol{U}_{\boldsymbol{a}}=0 & \text { on } \Gamma_{\text {in }} \cup \Gamma_{\text {lat }} \cup \Gamma \\
\sigma\left(\boldsymbol{U}_{\boldsymbol{a}}, p_{a}\right) \boldsymbol{n}+(\boldsymbol{U} \cdot \boldsymbol{n}) \boldsymbol{U}_{\boldsymbol{a}}=4 \nu \lambda_{\varepsilon} \varepsilon(\boldsymbol{U}) \boldsymbol{n} & \text { on } \Gamma_{\text {out }}
\end{aligned}\right.
$$

where $\mathcal{H}\left(\boldsymbol{U}, \boldsymbol{U}_{a}\right)=-\nu \Delta \boldsymbol{U}_{a}+(\nabla \boldsymbol{U})^{T} \boldsymbol{U}_{\boldsymbol{a}}-\nabla \boldsymbol{U}_{\boldsymbol{a}} \boldsymbol{U}+\lambda_{\varepsilon} 2 \nu \Delta \boldsymbol{U}$ and $C_{a}$ is the concentration of the adjoint state of $C$ defined as the solution of system:

$$
\left\{\begin{aligned}
-\mathcal{D} \Delta C_{a}-\boldsymbol{U} \cdot \nabla C_{a}+k C_{a}=0 & \text { in } \Omega \\
C_{a}=0 & \text { on } \Gamma_{\text {in }} \\
\frac{\partial C_{a}}{\partial \boldsymbol{n}}=0 & \text { on } \Gamma_{\text {lat }} \cup \Gamma \\
C_{a}(\boldsymbol{U} \cdot \boldsymbol{n})+\mathcal{D} \frac{\partial C_{a}}{\partial \boldsymbol{n}}=K_{\mathrm{BC}} & \text { on } \Gamma_{\text {out }}
\end{aligned}\right.
$$

In this work, $K_{\mathrm{BC}}$ is set to $3 \times 10^{-3} \mathrm{~mol} . \mathrm{m}^{-2} \cdot \mathrm{s}^{-1}$ in order to allow the fields $C$ and $C_{a}$ having the same order of magnitude. 


\subsection{Second case: heterogeneous reaction}

In this case, the same reasoning as in the first case is done and the shape gradient rewrites in the form:

$$
G\left(\Omega, \lambda_{\mathcal{V}}, \lambda_{\mathcal{E}}\right)=2 \nu\left(\varepsilon(\boldsymbol{U}): \varepsilon\left(\boldsymbol{U}_{\boldsymbol{a}}\right)-\lambda_{\mathcal{E}} \varepsilon(\boldsymbol{U}): \varepsilon(\boldsymbol{U})\right)+\frac{K_{\text {crit }}}{K_{\mathrm{BC}}} \mathcal{D} \frac{\partial C_{a}}{\partial \boldsymbol{n}} \frac{\partial C}{\partial \boldsymbol{n}}+\lambda_{\mathcal{V}}
$$

In Eq. (29), $\boldsymbol{U}_{\boldsymbol{a}}$ is the velocity of the adjoint state $\left(\boldsymbol{U}_{\boldsymbol{a}}, p_{a}\right)$ solution of Eqs. (27) and $C_{a}$ is the concentration of the adjoint state of $C$ defined as the solution of the following system:

$$
\left\{\begin{aligned}
-\mathcal{D} \Delta C_{a}-\boldsymbol{U} \cdot \nabla C_{a}=0 & \text { in } \Omega \\
C_{a}=0 & \text { on } \Gamma_{\text {in }} \cup \Gamma_{\text {lat }} \cup \Gamma \\
C_{a}(\boldsymbol{U} \cdot \boldsymbol{n})+\mathcal{D} \frac{\partial C_{a}}{\partial \boldsymbol{n}}=K_{\mathrm{BC}} & \text { on } \Gamma_{\text {out }}
\end{aligned}\right.
$$

\section{Implementation of the shape optimization algorithm}

Table 1 presents the optimization algorithm used to determine the optimal shape of the reactors. It is implemented within OpenFOAM (Weller et al., 1998) which is a free and open-source platform allowing to solve partial differential equations using $\mathrm{C}++$ programming language and the finite volume method. In order to link iterations to each other, a python library named pyFoam is used through its mesh utility "pyFoamMeshUtilityRunner.py".

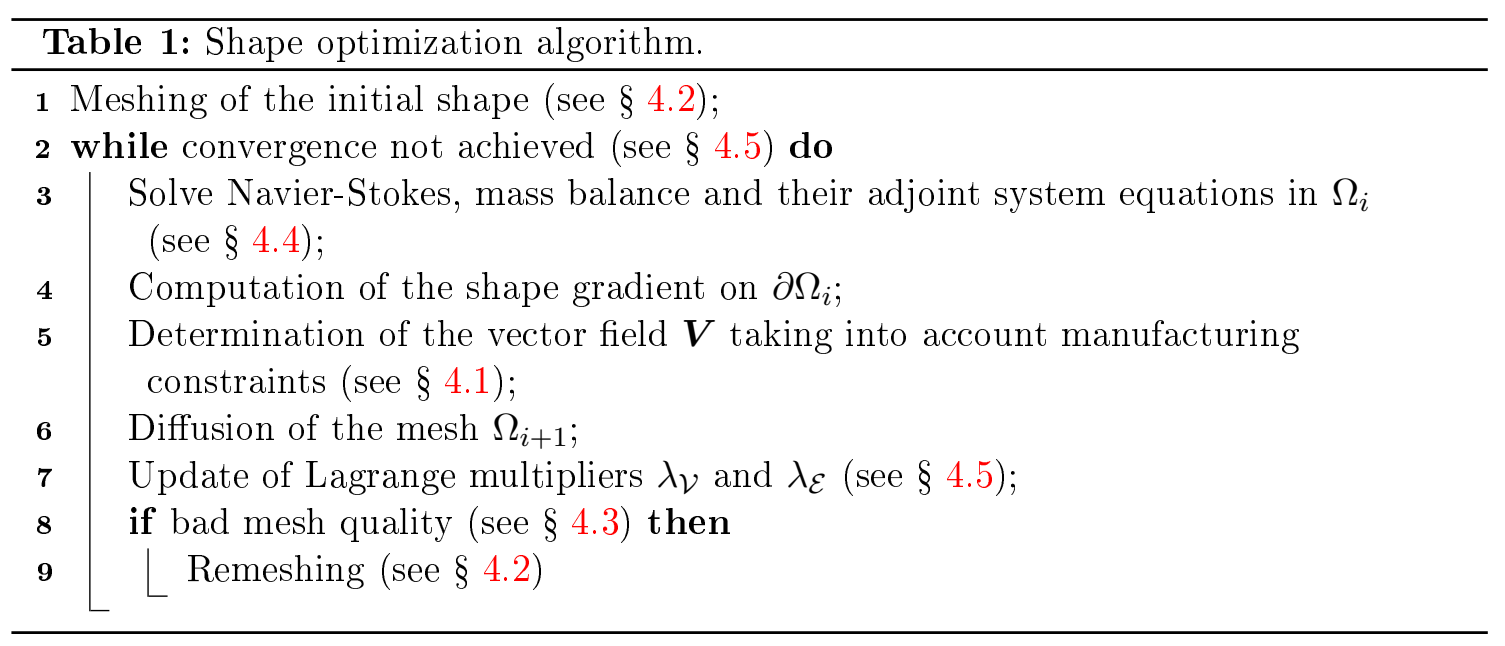

The next subsections will detail the algorithm and discuss in particular its accuracy.

\subsection{Mesh displacement}

The manufacturing constraints are treated by post-processing of the vector field $\boldsymbol{V}$ after its computation. 


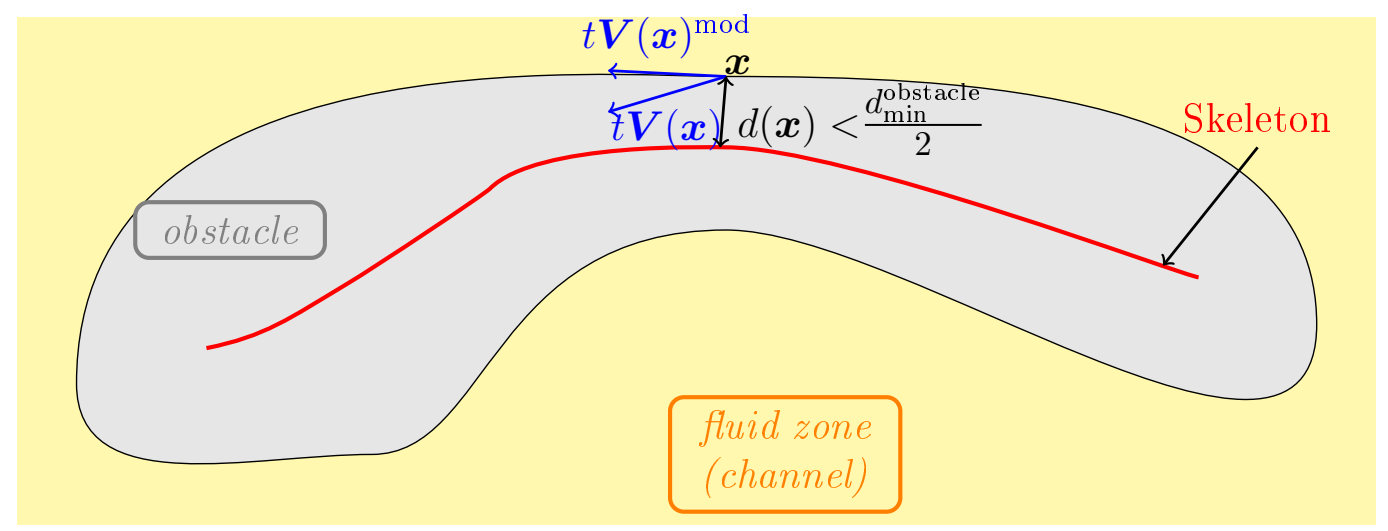

Figure 4: Illustration of the obstacle constraint treatment. $\boldsymbol{V}(\boldsymbol{x})$ and $\boldsymbol{V}(\boldsymbol{x})^{\text {mod }}$ are respectively the mesh displacement vector before and after modification at point $\boldsymbol{x}$.

\subsubsection{Obstacle thickness constraint}

This first manufacturing constraint is of inequality type and imposes a minimal value on the local thickness of obstacles $\left(d^{\text {obstacle }}>d_{\min }^{\text {obstacle }}\right)$. It is treated by a projection method in two main steps. The first one consists in determining the local thickness at point $\boldsymbol{x}$ and the second one in the projection of vector $\boldsymbol{V}(\boldsymbol{x})$.

The main difficulty of this treatment is to compute the thickness. Its estimation is trivial to the naked eye, however, its implementation is complex in practice. Indeed, choosing the right direction to quantify this length is not an easy task. In this work, a thin solid centered inside the obstacles, called skeleton, is constructed and the thickness of the obstacle is defined as the double of the distance between its boundary and its skeleton (Fig. 4). In two dimensions, the skeleton of an obstacle is the curve which is equidistant from the obstacle on each side (Fig. 5), its mathematical definition is as follows.

Definition 1 The skeleton of an obstacle is defined as the set of centers of the maximal ball totally included in the obstacle.

Definition $2 A$ ball $B_{1}$ included in a set $F$ is maximal if there is no ball $B_{2}$ also included in $F$ containing $B_{1}$ strictly in the sense of inclusion.

In this work, the skeleton construction is based on the work of Attali (1995) who built the skeleton of a shape from its Voronoi diagram, considering the shape as a discrete set of points. The Voronoi diagram of a set of points $E$ is determined from the Voronoi regions. In the $2 \mathrm{D}$ case, the Voronoi region of a point $\boldsymbol{x} \in E$ is defined as the area where points belonging to it are closest to point $\boldsymbol{x}$ than all other points of $E$ (Attali, 1995). It is mathematically defined below.

Definition 3 Let $X$ a subset of $\mathbb{R}^{d}$ and $P=\left\{\boldsymbol{P}_{\mathbf{1}}, \boldsymbol{P}_{\mathbf{2}}, \ldots, \boldsymbol{P}_{\boldsymbol{n}}\right\} \subset X$ a set of points. The Voronoi region $R_{k}$ of a point $\boldsymbol{P}_{\boldsymbol{k}}$ is defined as follows

$$
R_{k}=\left\{\boldsymbol{x} \in X \mid d\left(\boldsymbol{x}, \boldsymbol{P}_{\boldsymbol{k}}\right)<d\left(\boldsymbol{x}, \boldsymbol{P}_{\boldsymbol{j}}\right), \quad \forall j \neq k\right\}
$$




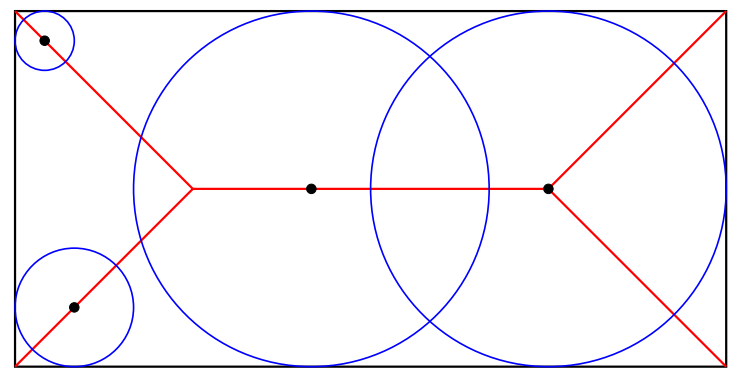

Figure 5: Schematic illustration of the skeleton of a rectangular object, the blue circles represent the maximal balls, the black dots their centers and the red line indicates the skeleton.

where $d\left(\boldsymbol{x}, \boldsymbol{P}_{\boldsymbol{k}}\right)$ is the distance between $\boldsymbol{P}_{\boldsymbol{k}}$ and $\boldsymbol{x}$.

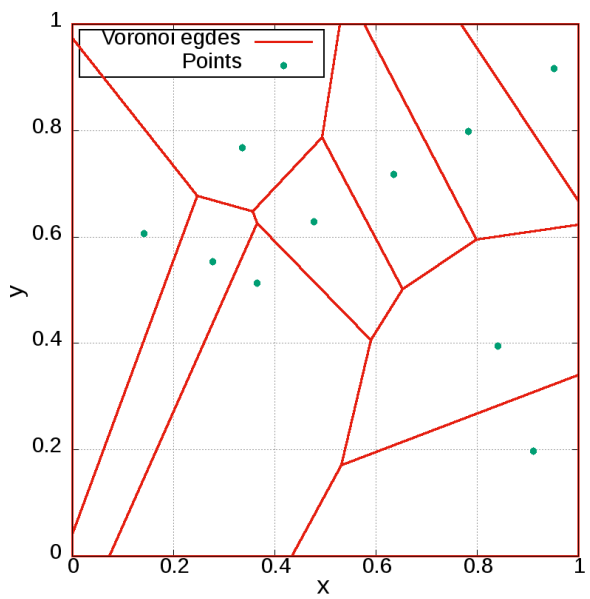

Figure 6: Voronoi diagram of a 10-point set.

Figure 6 illustrates the Voronoi diagram of a 10-point set. This diagram consists of two main elements, the vertices defined as the intersection of at least three Voronoi regions and the edges bounded by two vertices and defined as the boundary between two Voronoi regions. According to Attali (1995), the skeleton can be built from both vertices or edges. In this work, it is built from edges because this construction allows to obtain directly the skeleton contrary to the other way which requires a last step of linear interpolation between the vertices.

Once the Voronoi diagram built (Fig. 7(a)), it is reduced using two steps in order to obtain the final skeleton:

- Edges which are not completely included in the obstacle are removed (Fig. 7(b)).

- A second simplification is carried out using two criteria. (i) For all Voronoi vertices $s$, the first criterion is the minimum distance between $s$ and the obstacle, called $r(s)$. 
(ii) As previously mentionned, each point $s$ has at least three projections on the obstacle, called $p_{1}, p_{2}$ and $p_{3}$. The second criterion is defined as follow

$$
\alpha(s)=\max \left(\widehat{p_{1} s p_{2}}, \widehat{p_{1} s p_{3}}, \widehat{p_{2} s p_{3}}\right)
$$

The minimum values of $r(s)$ and $\alpha(s)$ are respectively $2 \times 10^{-4} \mathrm{~m}$ and $\frac{\pi}{2}$ (Fig. $7(\mathrm{c})$ ).

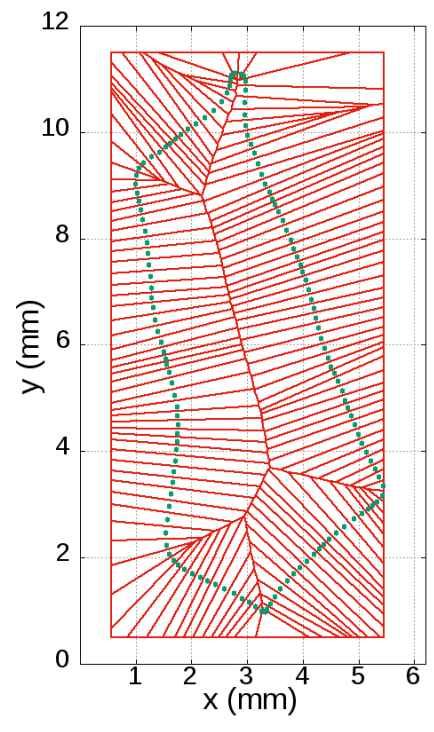

(a) Obstacle Voronoi diagram

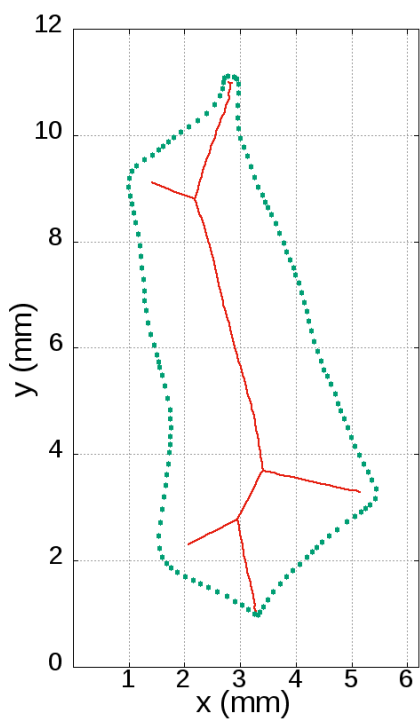

(b) First simplification

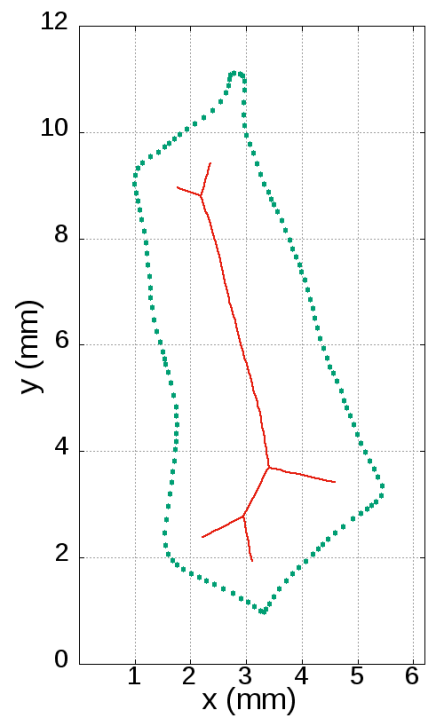

(c) Second simplification: skeleton

Figure 7: Simplification of the Voronoi diagram into the skeleton: green points denote obstacle points and red lines denote Voronoi diagram $(a)$ and the simplified skeleton $(b)$ and $(c)$.

The second step is carried out by means of a test on the minimum distance $d(\boldsymbol{x})$ between the free boundary and the skeleton. If this distance is lower than $\frac{d_{\min }^{\text {obstacle }}}{2}$ and $d(\boldsymbol{x})>d(\boldsymbol{x}+t \boldsymbol{V}(\boldsymbol{x}))$ then the vector $\boldsymbol{V}(\boldsymbol{x})$ is modified in order to be parallel to the skeleton (Fig. 4). The resulting vector is denoted $\boldsymbol{V}(\boldsymbol{x})^{\text {mod }}$.

\subsubsection{Constraint on channel width}

The other manufacturing constraint imposes a minimal value on the width of fluid channels. It is also treated in two main steps by a projection method. For each boundary point $\boldsymbol{x}$, the local channel width is first computed by looping over all boundary points belonging to another obstacle. The closest point belonging to another boundary is denoted $\boldsymbol{x}^{\text {near }}$. The second step consists in computing the inner product of vectors $\boldsymbol{x} \boldsymbol{x}^{\text {near }}$ and $\boldsymbol{V}(\boldsymbol{x})$. If this scalar product is positive and $d(\boldsymbol{x})=\left\|\boldsymbol{x} \boldsymbol{x}^{\text {near }}\right\|<d_{\text {min }}^{\text {channel, }}$, then the vector $\boldsymbol{V}(\boldsymbol{x})$ is modified in order to be orthogonal to $\boldsymbol{x} \boldsymbol{x}^{\text {near }}$. Figure 8 shows an illustration of the channel constraint treatment. 


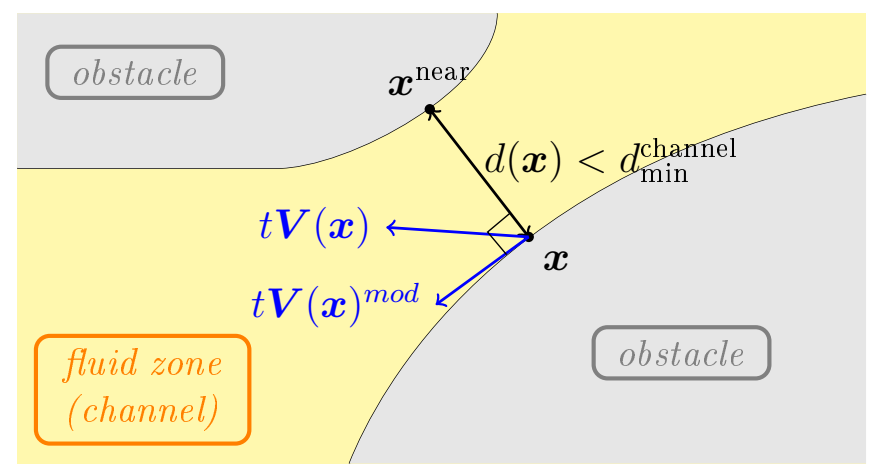

Figure 8: Illustration of the treatment of the constraint on the channel width. $\boldsymbol{V}(\boldsymbol{x})$ and $\boldsymbol{V}(\boldsymbol{x})^{\text {mod }}$ are, respectively, the mesh displacement vector before and after modification at point $\boldsymbol{x}$.

\subsection{Meshing and remeshing of the domain}

OpenFOAM solves the PDEs using the finite volume method which requires a mesh generation in order to discretize the governing equations. The meshing step is important because the quality of the solution depends strongly on the mesh quality. In this work, the meshing and the remeshing are carried out using cfMesh, an open-source library for automatic mesh generation. Then, the mesh quality is improved using snappyHexMesh, a mesh generator utility supplied by OpenFOAM. The resulting mesh is composed of 50,000 to 120,000 computational cells depending on the case. Figure 9 presents the grid independence test carried out for both cases. It shows the evolution of the outlet concentration of the reactant versus the number of cells in the mesh and validates the independence of the computed solution with respect to the used mesh density.

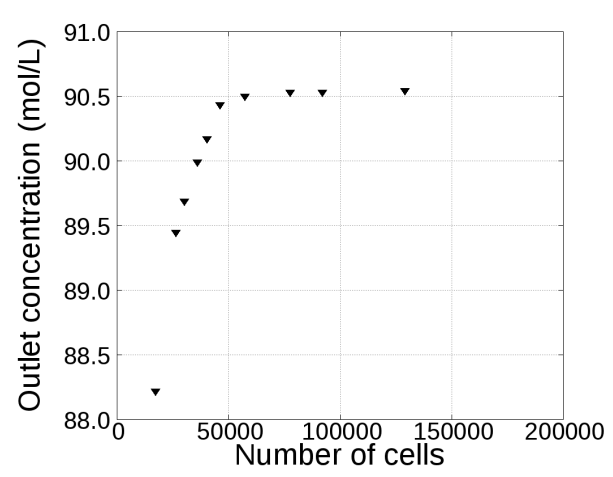

(a) (HR) case

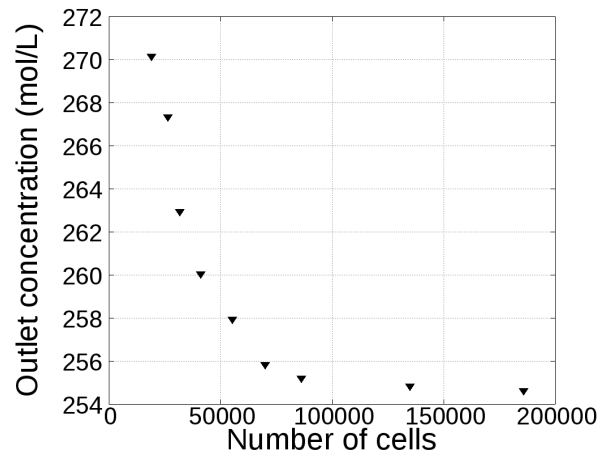

(b) (SR) case

Figure 9: Grid independence test - Outlet reactant concentration versus the number of cells in the mesh.

The shape gradient is a function defined on the free boundary $\Gamma$ and involves first and second order derivatives of the different variables (see Eqs. (26) and (29)). Thus, 


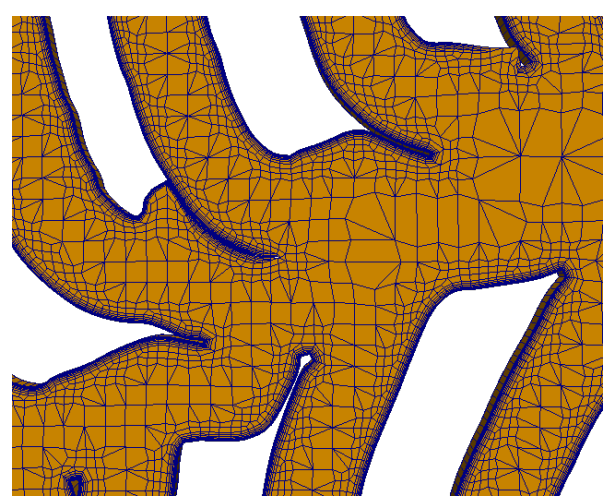

(a) Example of obstacles configuration during the optimization process

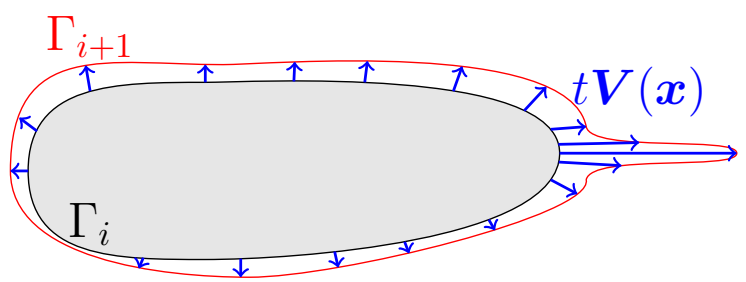

(b) Formation of tails at free boundary ends

Figure 10: Illustration of (a) the potential configuration of some obstacles, (b) the formation process of tails at free boundary ends.

the neighborhood of the boundary has to be modeled with accuracy, therefore, two layer meshes are added at boundary $\Gamma$ for the homogeneous reaction case. For the (SR) case, 5 layer meshes are added at the reaction boundaries in order to improve the modeling of mass transfer diffusion in the near-wall region.

Figure 10(a) illustrates the configuration that obstacles may present during the optimization process. According to the figure, "tails" may appear at the obstacle ends. This phenomenon was expected for the heterogeneous reaction case since the "tails" appearance allows to increase the reactive surface without much change in reactor volume. However, those "tails" are not manufacturable using Stratoconception ${ }^{\circledR}$ printing process due to their small local thickness. The projection treatment of manufacturing constraints (section 4.1) does not prevent the formation of these "tails" illustrated in Fig. 10(b). In order to ensure the manufacturability of the object, the "tails" formed during the optimization process are removed by moving, once detected, all boundary points belonging to the "tails" at the center of their extreme points just before performing the remeshing step.

\subsection{Mesh quality}

At each iteration, the mesh quality is checked to know whether the volume discretization will impact the quality of the PDEs solution. This quality verification is carried out through three criteria often used in CFD area. Those criteria, illustrated in Fig. 11, are (Holzinger, 2015):

- The aspect ratio, defined in two dimensions as the ratio of the biggest to the smallest length of a cell. It is expressed by the ratio $\frac{l}{s}$ (Fig. 11).

- The mesh non-orthogonality, defined as the angle between the vector connecting the cell centers of two adjacent cells and the normal of the common face. In Fig. 11, it is defined by angle $\alpha=\arccos \left(\frac{\boldsymbol{A}_{\boldsymbol{i}} \cdot \boldsymbol{C}_{\boldsymbol{i}}}{\left|\boldsymbol{A}_{\boldsymbol{i}}\right|\left|\boldsymbol{C}_{\boldsymbol{i}}\right|}\right)$. 
- The face skewness, defined by the ratio $\frac{\left|\boldsymbol{d}_{\boldsymbol{i}}\right|}{\left|\boldsymbol{C}_{\boldsymbol{i}}\right|}$ (see Fig. 11), where $\left|\boldsymbol{d}_{\boldsymbol{i}}\right|$ is the distance between the intersection of the line connecting the adjacent cell centers and their common face, and the center of this face, $\left|\boldsymbol{C}_{\boldsymbol{i}}\right|$ is the distance between the centers of considered cells.

If the maximum value of the aspect ratio is higher than 20 , the mesh non-orthogonality is higher than $65^{\circ}$ or the face skewness is higher than 3.8, the shape is remeshed. Those upper bounds have been chosen because OpenFOAM checks the mesh quality computing (among others) the maximal values of the face skewness and the non-orthogonality of the mesh. If those values are respectively higher than 4 and $70^{\circ}$, the mesh quality is not validated. Slightly lower values have been chosen for the mesh non-orthogonality and the face skewness to ensure that the maximum values defined by OpenFOAM are not exceeded after each mesh displacement. The choice of the upper bound for the aspect ratio is based on its values in the initial shapes. Depending on the case, the maximum aspect ratio in the initial mesh is between 8 and 15, therefore the upper bound for this criterion has been set to 20 . However, this choice is not critical because the majority of the remeshing process launches is due to a violation of the criteria on non-orthogonality and skewness of the faces.

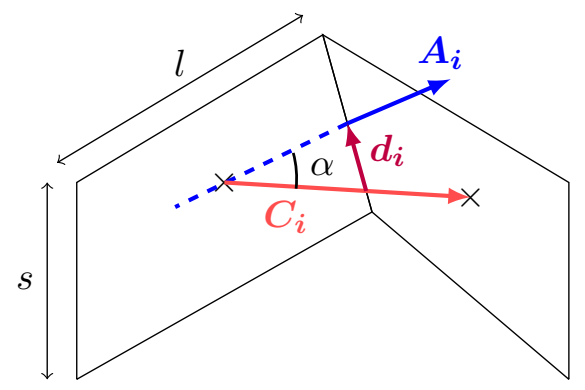

Figure 11: Illustration of the mesh quality criteria.

\subsection{Boundary conditions approximation}

The pressure-velocity couplings present in the Navier-Stokes Eqs. (1) and its adjoint system equations (27) are numerically solved using SIMPLE algorithm (Patankar, 1980).

The three outlet boundary conditions of the adjoint systems $((28 \mathrm{~d}),(30 \mathrm{c})$ and $(27 \mathrm{~d}))$ are not usual boundary conditions and must be implemented within OpenFOAM using the following approximations:

$$
\begin{gathered}
\left(\nabla \boldsymbol{U}_{\boldsymbol{a}}\right)^{T} \boldsymbol{n} \approx \frac{\boldsymbol{U}_{\boldsymbol{a}}^{\text {patch }}-\boldsymbol{U}_{\boldsymbol{a}}^{\text {intern }}}{\delta} \\
\nabla C_{a} \boldsymbol{n} \approx \frac{C_{a}^{\text {patch }}-C_{a}^{\text {intern }}}{\delta}
\end{gathered}
$$

which allows to rewrite the boundary conditions as follows:

$$
\boldsymbol{U}_{\boldsymbol{a}}{ }^{\mathrm{patch}} \approx \frac{\nu \delta^{-1} \boldsymbol{U}_{\boldsymbol{a}}^{\text {intern }}+4 \nu \lambda_{\varepsilon} \varepsilon(\boldsymbol{U}) \boldsymbol{n}+p_{a} \boldsymbol{n}-\nu \nabla \boldsymbol{U}_{\boldsymbol{a}} \boldsymbol{n}}{\nu \delta^{-1}+(\boldsymbol{U} \cdot \boldsymbol{n})}
$$


Table 2: Simulation parameters.

\begin{tabular}{cccc}
\hline Parameters & Values & Units & Equations \\
\hline$\nu$ & $10^{-6}$ & $\mathrm{~m}^{2} \cdot \mathrm{s}^{-1}$ & $(1 \mathrm{a}),(26),(27)$ and $(29)$ \\
$U_{\text {in }}$ & $10^{-2}$ & $\mathrm{~m}^{-\mathrm{s}^{-1}}$ & $(1 \mathrm{c})$ \\
$\mathcal{D}$ & $10^{-9}$ & $\mathrm{~m}^{2} \cdot \mathrm{s}^{-1}$ & $(26),(28 \mathrm{a}),(29)$ and $(30 \mathrm{a})$ \\
$k$ & $10^{-2}$ & $\mathrm{~s}^{-1}$ & $(5 \mathrm{a})$ \\
$\gamma$ & $10^{-4}$ & $\mathrm{~m}^{-2}$ & $(24 \mathrm{a})$ and $(24 \mathrm{c})$ \\
$K_{\mathrm{BC}}$ & $3 \times 10^{-3}$ & $\mathrm{~mol}^{-2} \mathrm{~m}^{-1}$ & $(26),(27 \mathrm{~d}),(28 \mathrm{~d}),(29)$ and $(30 \mathrm{c})$ \\
$K_{\text {crit }}$ & $3 \times 10^{-5}$ & $\mathrm{~m}^{6} \cdot \mathrm{s}^{-3} \cdot \mathrm{mol}^{-1}$ & $(10),(26)$ and $(29)$ \\
\hline
\end{tabular}

$$
C_{a}^{\text {patch }} \approx \frac{\nu \delta^{-1} C_{a}^{\text {intern }}+K_{\mathrm{BC}}}{\nu \delta^{-1}+(\boldsymbol{U} \cdot \boldsymbol{n})}
$$

where $\delta$ is the distance between the boundary and the internal adjacent cell center, $\boldsymbol{U}_{\boldsymbol{a}}{ }^{\text {patch }}$ and $C_{a}^{\text {patch }}$ represent respectively the adjoint velocity and the adjoint concentration at the boundary, and $\boldsymbol{U}_{\boldsymbol{a}}$ intern and $C_{a}^{\text {intern }}$ their values in the internal adjacent cell center. The implementation of these boundary conditions within OpenFOAM is presented with further details in Courtais (2021).

\subsection{Lagrange multipliers update and convergence of the algorithm}

In this work, the optimization algorithm used is based on the Uzawa method which consists in the determination of the mesh displacement at each iteration considering the Lagrange multipliers $\lambda_{\mathcal{V}}$ and $\lambda_{\mathcal{E}}$ constant. Once the mesh diffusion done, the Lagrange multipliers are updated according the following equations:

$$
\begin{gathered}
\lambda_{\mathcal{V}}^{k+1}=\lambda_{\mathcal{V}}^{k}+\beta_{\mathcal{V}} C_{\mathcal{V}}(\Omega) \\
\lambda_{\mathcal{E}}^{k+1}=\max \left(0, \lambda_{\mathcal{E}}^{k}+\beta_{\mathcal{E}} C_{\mathcal{E}}(\Omega)\right)
\end{gathered}
$$

where $\beta_{\mathcal{v}}$ and $\beta_{\mathcal{E}}$ are small positive parameters. The formulation of relations (36) and (37) are different because the volume constraint is of equality type while the energy constraint is an inequality one (see Karush-Kuhn-Tucker's dual feasibility condition (Karush, 2014; Kuhn and Tucker, 2014)). This explains why $\lambda_{\mathcal{V}}$ may be negative while $\lambda_{\mathcal{E}}$ cannot.

Finally, the convergence of the algorithm is carried out through the computation of the coefficient of variation (or relative standard deviation) of the last 100 Lagrangian values. If this ratio is lower than $10^{-4}$, the convergence is achieved.

\section{Main results}

This section is devoted to the presentation of numerical results obtained using the algorithm described in the previous section. It consists of two subsections where the optimization 
(a)

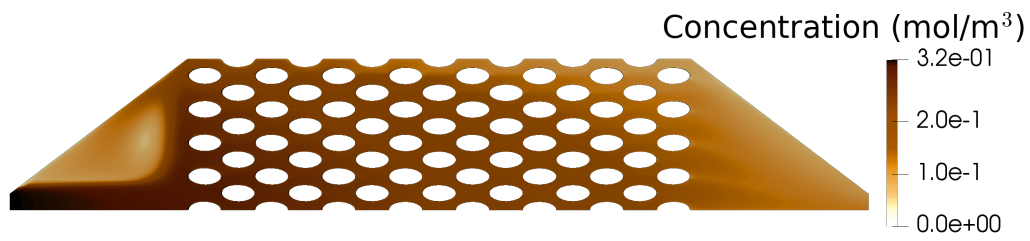

(b)

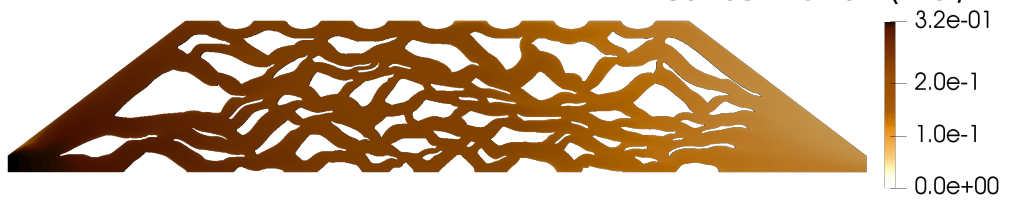

(c)

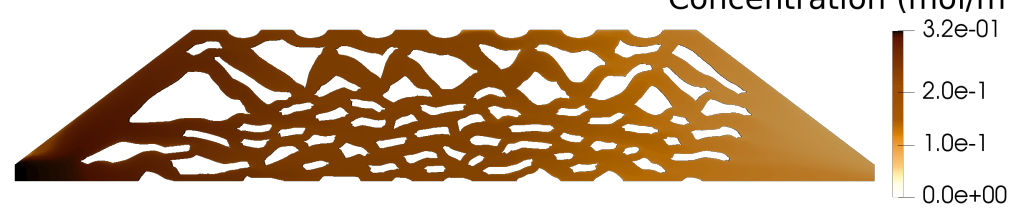

(d)

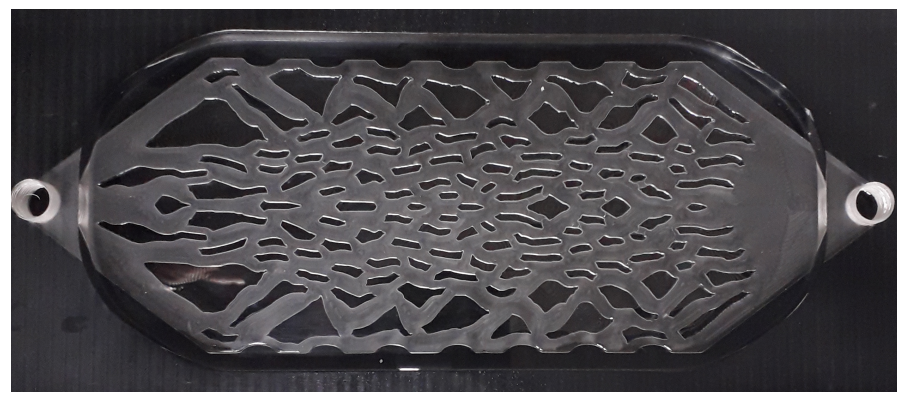

Figure 12: Homogenous reaction case: initial design of the fixed-bed reactor (a), optimized shape without manufacturing constraint (b), optimized shape with manufacturing constraint (c), optimal shape manufactured by means of Stratoconception ${ }^{\circledR}$ process (d).

results for the cases (HR) and (SR) are presented. Except for the kinetic constant $k$, the simulation parameters are the same for both cases and are shown in Table 2.

\section{$5.1 \quad$ Homogeneous reaction}

Figures 12(a) and 12(b) show the concentration profiles of the reactant in the initial design and in the optimal shape (without considering the manufacturing constraints) of the reactor. As can be seen, a stagnation zone appears at the inlet of the initial shape (light area in Figure 12(a)). It corresponds to a region of the reactor where the reactant concentration is low resulting in low reaction rates. Consequently, this stagnation volume is almost useless for the conversion. In the optimal shape without manufacturing constraints, the stagnation zone has disappeared. However, this reactor configuration cannot be manufactured using Stratoconception ${ }^{\circledR}$ process due to too thin obstacles and some very small channel widths.

The optimization is then carried out taking into account the manufacturing constraints 

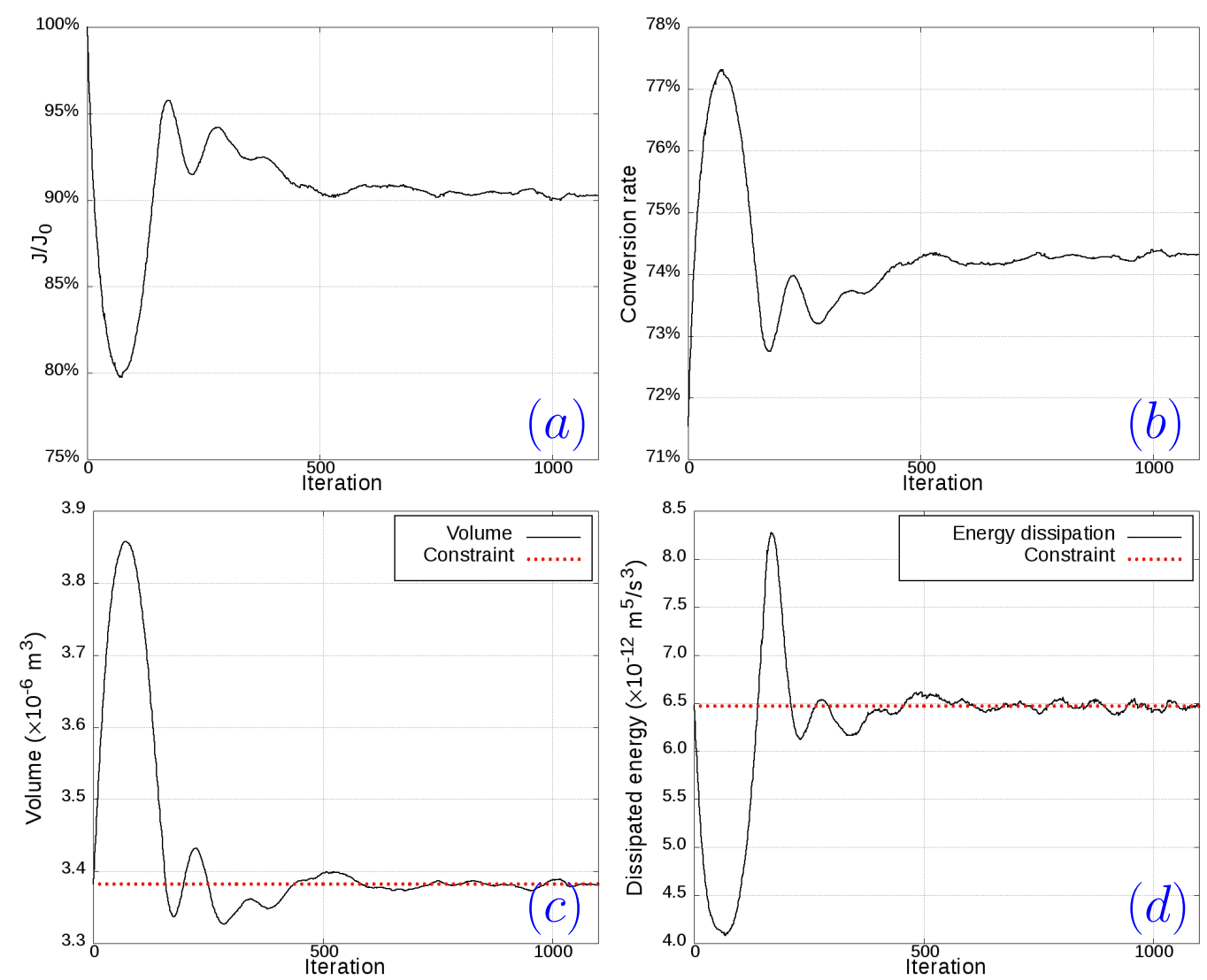

Figure 13: Performances of the optimization process of the reactor satisfying the manufacturing constraints (Fig. 12(c)), (a) ratio $\frac{J}{J_{0}},(b)$ conversion rate, $(c)$ volume constraint, $(d)$ energy dissipation constraint, versus iterations.

and the resulting shape is displayed in Fig. 12(c). Similarly, the stagnation zone present in the initial shape has disappeared and the size of obstacles and channels of the optimized shape are such that they can be built by means of Stratoconception ${ }^{\circledR}$ process. The manufactured optimal shape of the reactor is shown in Figure 12(d).

Figure 13 shows the convergence history of the optimization process. The energy and volume constraints are both satisfied at convergence and it is interesting to note that the inequality constraint on the energy dissipated is active. It may therefore be interesting either to increase the maximum value associated to this constraint in order to obtain a better conversion rate, or to perform multi-objective optimization of the reactor considering two criteria $(i)$ the conversion rate and $(i i)$ the energy dissipation. Figure 13 also shows a decrease of almost $10 \%$ in the reactant concentration at the reactor outlet, which leads to an improvement in the conversion rate of $2.7 \%$ ( $71.5 \%$ versus $74.2 \%$ ). This improvement can be explained by two main reasons. The first one is the disappearance of the stagnation zone and the second one is the better homogeneity of the liquid flow in the reactor. This 


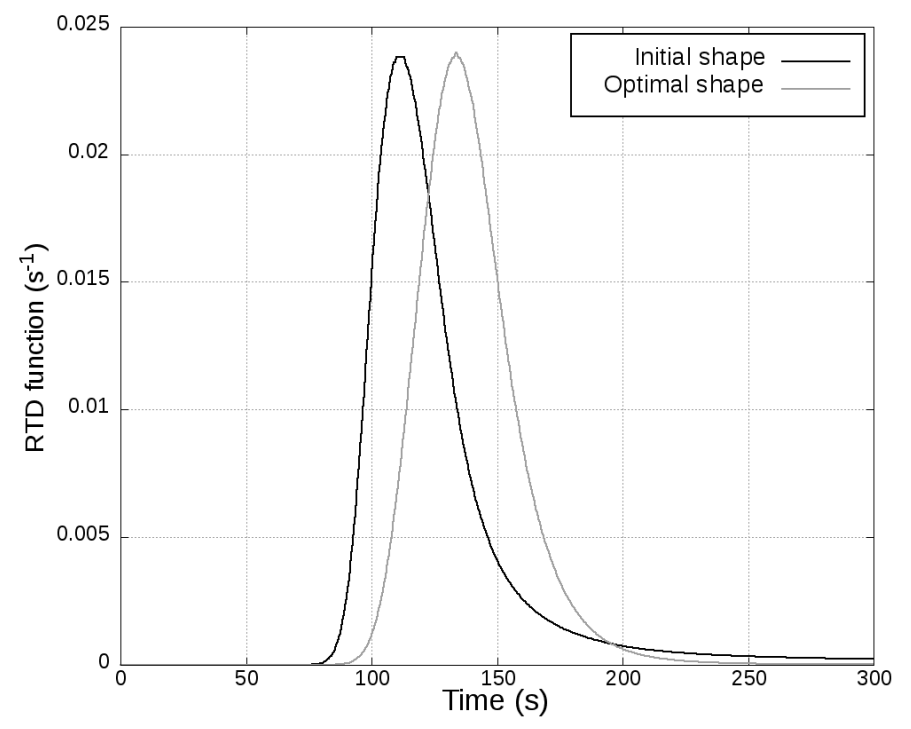

Figure 14: Residence time distribution of initial and optimal reactors.

better homogeneity is shown by the residence time distributions (RTDs) presented in Fig. 14. These RTDs have been determined numerically by imposing a concentration pulse at the inlet boundary once the fluid flow is developed. The reactor response to this pulse is then examined by determining the variation of the concentration at the outlet over time. The RTDs exploitation is then carried out by means of the method of moments which consists of determining the zeroth $(i=0)$, first $(i=1)$ and second $(i=2)$ order moments given by (Levenspiel, 1999):

$$
\mu_{i}=\int_{0}^{\infty} t^{i} E(t) d t
$$

where $E(t)$ is the RTD function. The standard deviation of the RTDs is 3.5 times lower in the optimized reactor than in the initial one (Table 3 ) which is mainly explained by the disappearance of the stagnation zone. Indeed, Fig. 14 shows that the RTD function hits almost zero in the optimal shape after $250 \mathrm{~s}$ which is not the case in the initial shape. The disappearance of the stagnation zone is also highlighted regarding the first order moment since the mean residence time is slightly lower for initial reactor shape.

Table 3: RTDs parameters - (HR) case.

\begin{tabular}{|c|c|c|c|c|}
\hline \multirow{2}{*}{$\begin{array}{l}\text { Reactor } \\
\text { shape }\end{array}$} & \multicolumn{3}{|c|}{ Moment of order } & \multirow{2}{*}{$\begin{array}{l}\text { Standard deviation } \\
\quad\left(\sigma=\sqrt{\mu_{2}-\mu_{1}^{2}}\right)\end{array}$} \\
\hline & Zero & One (mean) & Two & \\
\hline Initial & 1 & $134 \mathrm{~s}$ & $23284 \mathrm{~s}^{2}$ & $73 \mathrm{~s}$ \\
\hline Optimal & 1 & $139 \mathrm{~s}$ & $19868 \mathrm{~s}^{2}$ & $21 \mathrm{~s}$ \\
\hline
\end{tabular}

The deviation of the optimized reactor performances from the ideal plug flow reactor 
is then analyzed. Indeed, the conversion rate in a steady-state plug flow reactor where a first order homogeneous reaction occurs is given by (Villermaux, 1993):

$$
X_{A}=1-e^{-k \tau}
$$

where $\tau=\frac{\mathcal{V}\left(\Omega_{0}\right)}{Q}$ is the hydraulic residence time with $Q$ representing the fluid flow rate. In this case, the deviation from the flug flow reactor is small since the conversion rate of the ideal reactor is $74.6 \%$ (versus $74.2 \%$ for the optimized reactor). Thus, this validates the performance of the optimization procedure since it minimizes the deviation from the ideal reactor. In this case, the packing acts as a static mixer and the optimization procedure aims at improving the homogeneity of the fluid flow in order to reduce the deviation of the reactor from the ideal plug flow reactor.

The assumption of laminar flow is checked computing the channel Reynolds number. In this case, the particle Reynolds number is meaningless because obstacles have different dimensions. The channel Reynolds number is expressed by the following equation:

$$
\operatorname{Re}_{\mathrm{c}}=\frac{u d}{\nu}
$$

where $u$ is the fluid average velocity which flows inside the channel, $d$ the channel width and $\nu$ the fluid kinematic viscosity. In the optimized configuration 12(c) the Reynolds number $\mathrm{Re}_{\mathrm{c}}$ does not exceed 16 which validates the assumption of laminar flow.

The presence of local minima is analyzed by starting the optimization procedure from a slightly modified initial shape. Fig. 15 presents the optimal reactor shapes determined when: (a) the initial obstacles are enlarged (i.e. the semi-axes are increased by $0.5 \mathrm{~mm}$ ), and (b) the obstacles are translated by $3 \mathrm{~mm}$ towards the reactor inlet. As can be seen, the optimized reactors are slightly different from each other and from the one displayed in Fig. 12(c) showing the presence of local minima. However, since the conversion rate is improved in the same proportions (by $2.4 \%$ for the three optimal shapes), these 3 optimal reactors are in the same level of performance.

The impact of the kinetic constant on the determined optimized shape is then studied. Two additional optimizations when the kinetic constant is equal to (a) $5 \times 10^{-2} \mathrm{~s}^{-1}$, and to (b) $2 \times 10^{-3} \mathrm{~s}^{-1}$ have been carried out (Fig. 16). Both optimal shapes are not significantly different from the optimized shapes presented on Figs. 12(c) and 15. It was expected because, as previously pointed out for the (HR) case, the aim of the optimization procedure is to find the configuration of the packing which improves as much as possible the fluid flow homogeneity in the reactor. Therefore, since the kinetic constant does not influence the fluid flow in the reactor, it has no impact on the optimized configurations of the reactor. In the present case, the optimal shapes (Fig. 16) are slightly different since the algorithm converged towards different local minima.

Another optimization has been carried out by increasing the fluid kinematic viscosity by a factor of 10 in order to study the sensitivity of the optimal shape with respect to Reynolds number. The hydrodynamics in the reactor is therefore modified and the initial and optimal shapes of the reactor are shown on Fig. 17. The stagnation zone present in the initial shape of the reactor (a) is much less pronounced in this case due to the viscosity 
(a)

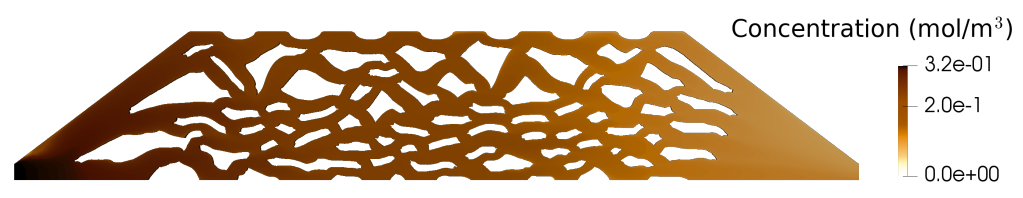

(b)

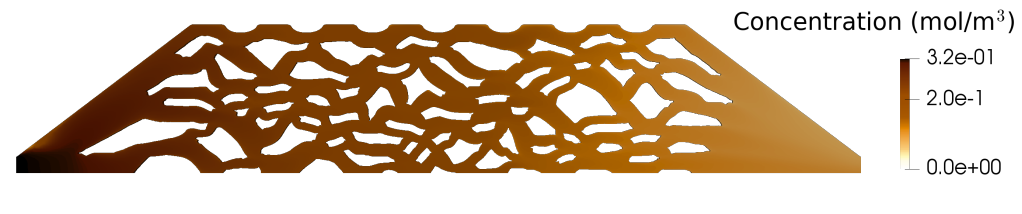

$\mathrm{O} \quad \mathrm{y}$

Figure 15: Optimal reactors when initial shape is slightly perturbed: (a) the semi-axes of initial obstacles are increased by $0.5 \mathrm{~mm}$ and (b) the obstacles are translated by $3 \mathrm{~mm}$ towards the reactor inlet

(a)
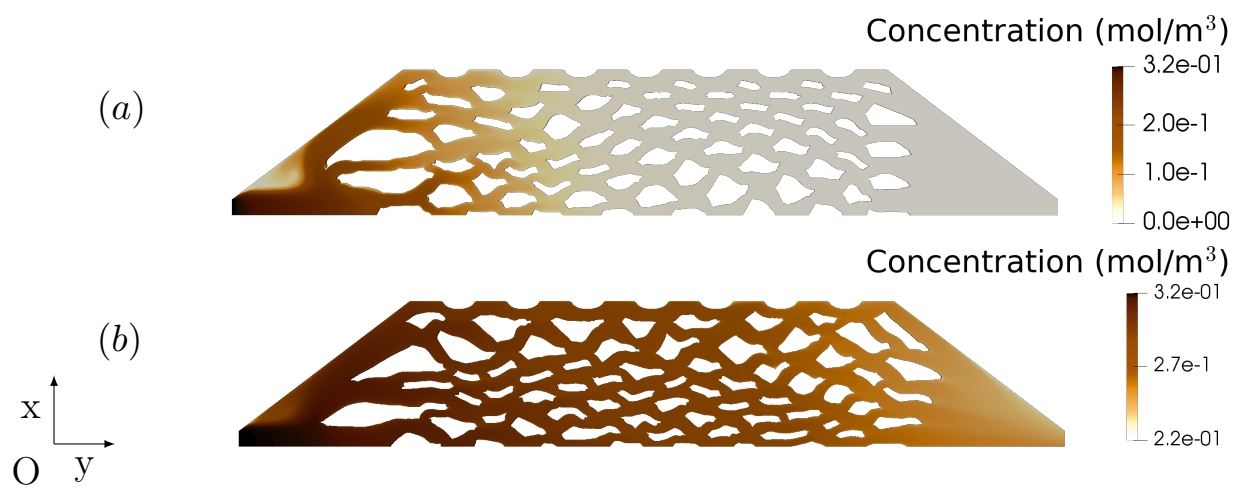

Figure 16: Optimal reactors when the kinetic constant is: (a) multiplied by 5 and (b) divided by 5 .

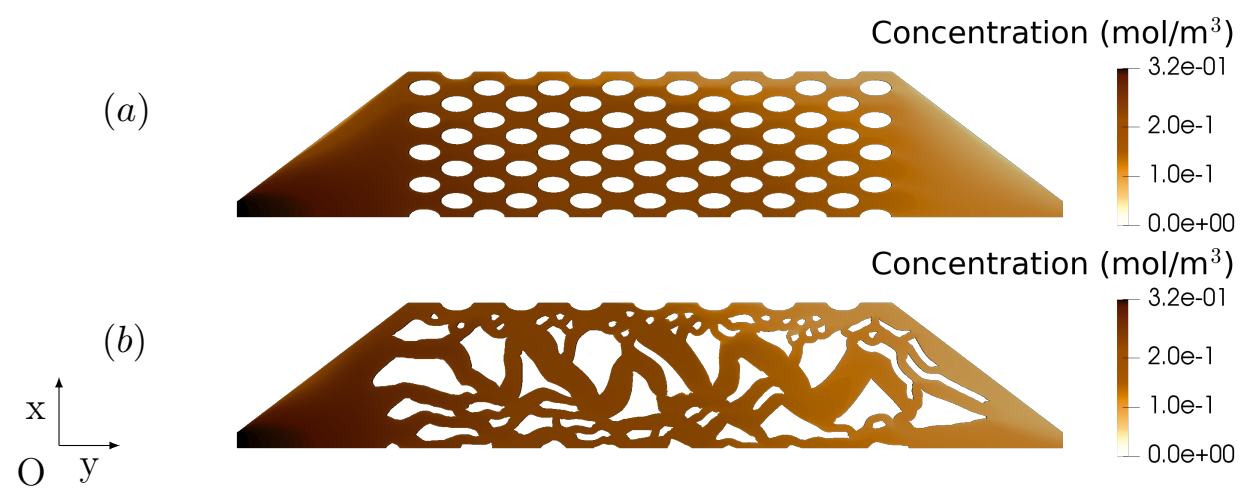

Figure 17: Initial (a) and optimized (b) reactors when the fluid kinematic viscosity is multiplied by 10 . 
(a)

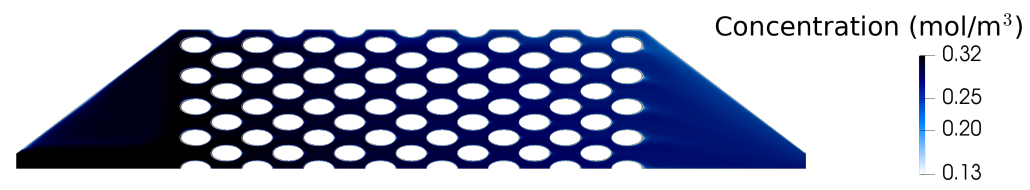

(b)

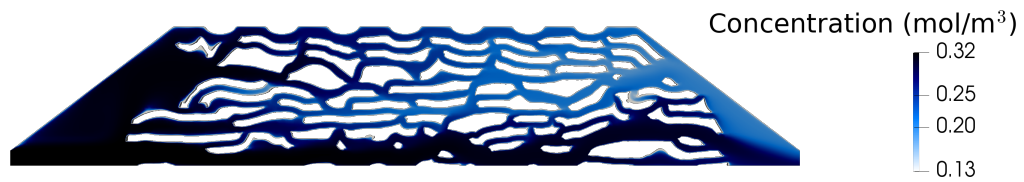

(c)
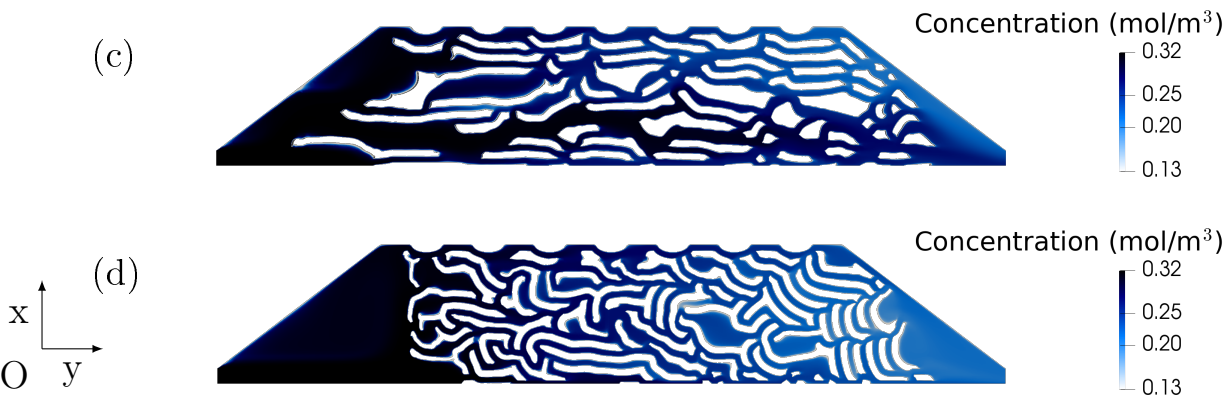

Figure 18: Surface reaction case: initial design of the fixed-bed reactor (a), optimized shapes subjected to iso-energy constraint (b), subjected to 1.4 times initial energy constraint (c) and without energy constraint (d).

increase. Therefore, the optimization process does not modify the packing structure in order to break/remove this stagnation zone in contrast to the previous optimizations. Consequently, as we can see, the optimal shape displayed on Fig 17(b) is quite different from the others presented on Figs. 12, 15 and 16. Finally, the optimization performances are similar to the previous ones since the conversion rate of the optimized reactor (b) is about $74.2 \%$ under manufacturing, volume and energy constraints.

\subsection{Heterogeneous reaction}

Figure 18 presents the numerical results of the reactor optimization. In particular, figures 18(a) and 18(b) illustrate the concentration profiles in the initial and optimal shapes. Here, the optimization allows a decrease of the performance index of almost $6 \%$ which results in $4.7 \%$ improvement in the reactor conversion rate (19.6\% versus $24.3 \%)$. It is mainly explained by the $50 \%$ increase of the reaction surface (Fig. 19(a)). However, the configuration (b) of the reactor presents poor homogeneity in the liquid flow. Indeed, the wall surface increase leads to an increase of the energy dissipation due to wall shear stress. Thus, the reactor exhibits channeling in its center (close to the axis of symmetry) in order to satisfy the energy constraint.

Two more optimizations with and without energy constraint are carried out. In the case with constraint, the upper bound of the constraint is multiplied by a factor of 1.4 to 

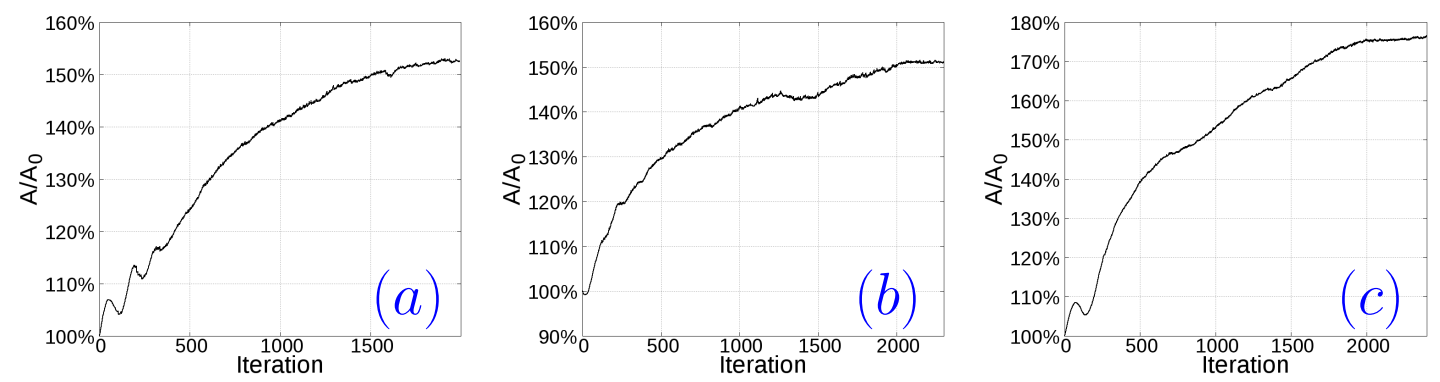

Figure 19: Evolution of the specific surface area over iterations: optimization under isoenergy constraint (a), optimization under energy constraint (1.4 times initial energy losses) (b), and optimization without energy constraint (c).
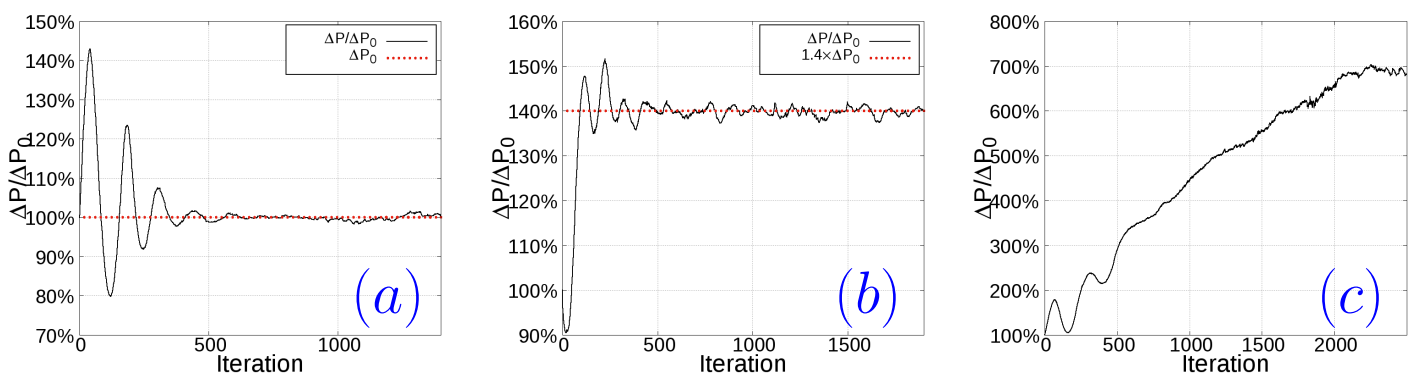

Figure 20: Evolution of the pressure drops in the reactor over iterations: optimization under iso-energy constraint (a), optimization under energy constraint (1.4 times initial energy losses) (b), and optimization without energy constraint (c).

analyze its effect on the fluid homogeneity in the reactor. The resulting optimal shapes are displayed in Figs. 18(c) and 18(d). They show that the higher the upper bound of energy constraint is, the more homogeneous the fluid flow becomes and the more the channeling in the reactor reduces. The improvement of the fluid homogeneity, the increase of the reactive surface area (Figs. 19(b) and 19(c)) and the disappearance of the channeling lead to an increase in the performances of optimal reactors. Indeed, the reactant concentration at the reactor outlet has decreased by $8 \%$ and $20 \%$ for the shapes $18(\mathrm{c})$ and $18(\mathrm{~d})$, respectively, which improves the conversion rate by $6.4 \%$ and $16 \%$. However, these improvements of the conversion rate inevitably lead to an increase of the pressure drops in both reactors by a factor of 1.4 and 7 respectively (Fig. 20). Figure 21 presents the RTDs of the three optimal reactors (with their associated description numbers gathered in Table 4) and shows the reduction of the channeling in the reactor when the upper bound of the energy constraint is increased. The distribution associated to the optimization with iso-energy constraint exhibits two peaks, the first of which corresponds to the channeling. The distribution associated to shape 18(c) presents also two peaks but the one referring to the channeling 


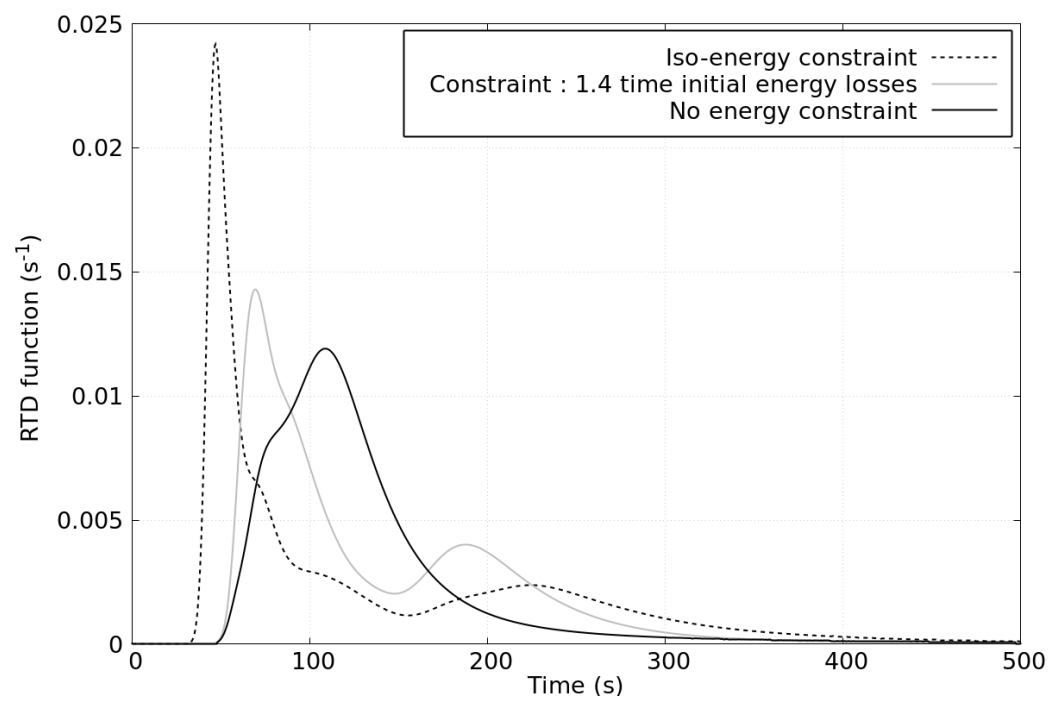

Figure 21: Residence time distribution of optimized reactors.

Table 4: RTDs parameters - (SR) case.

\begin{tabular}{ccccc}
\hline $\begin{array}{c}\text { Optimal } \\
\text { shape }\end{array}$ & Zeroth & First (mean) & Second & $\begin{array}{c}\text { Standard deviation } \\
\left(\sigma=\sqrt{\mu_{2}-\mu_{1}^{2}}\right)\end{array}$ \\
\hline Iso-energy constraint & 1 & $139 \mathrm{~s}$ & $32209 \mathrm{~s}^{2}$ & $114 \mathrm{~s}$ \\
1.4 times initial energy & 1 & $138 \mathrm{~s}$ & $26023 \mathrm{~s}^{2}$ & $84 \mathrm{~s}$ \\
No energy constraint & 1 & $138 \mathrm{~s}$ & $25902 \mathrm{~s}^{2}$ & $83 \mathrm{~s}$ \\
\hline
\end{tabular}

is less important than for the shape 18(b). In the reactor 18(d), the RTD shows that the channeling has disappeared. To summarize, in this case, the optimization procedure aims at increasing as much as possible the reactive surface (Fig. 19) and at improving the fluid flow homogeneity under pressure drops constraint.

For the three optimal reactors, channel Reynolds numbers have been computed and do not exceed 67, 48 and 17, respectively, for shapes 18(b), 18(c) and 18(d) which validates the assumption of the flow regime.

The sensitivity of the optimal shape with respect to the inlet flow velocity is finally investigated for the (SR) case. Two additional optimizations have been performed when the inlet flow velocity is reduced (a) by a factor of 2, and (b) by a factor of 10 (Fig. 22). As can be seen, the optimized shapes are significantly different from each other and from the one displayed on Fig. 18(b) leading to the conclusion that the optimized shape of the reactor may depend on the operating conditions such as the inlet flow rate. Table 5 presents the conversion rate of these three reactors depending on the inlet flow velocity imposed, and it supports the stated conclusion. Indeed, for $U_{\text {in }}=0.001 \mathrm{~m} . \mathrm{s}^{-1}$ (second column of the table), the reactor that leads to the highest conversion rate is the one displayed on 


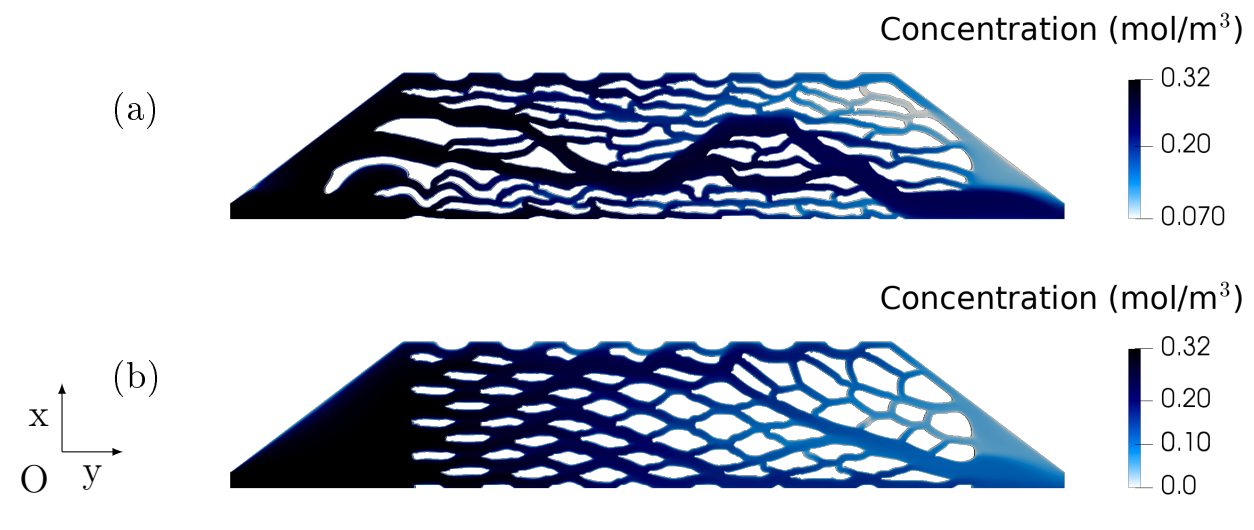

Figure 22: Surface reaction case: optimized design of the fixed-bed reactor when the inlet flow velocity is (a) $U_{\text {in }}=0.005 \mathrm{~m} . \mathrm{s}^{-1}$, (b) $U_{\text {in }}=0.001 \mathrm{~m} . \mathrm{s}^{-1}$.

Table 5: Conversion rate of optimal shapes depending on the inlet flow velocity (expressed in $\left.\mathrm{m} . \mathrm{s}^{-1}\right)-(\mathrm{SR})$ case.

\begin{tabular}{|l|ccc|}
\hline & $U_{\text {in }}=0.001$ & $U_{\text {in }}=0.005$ & $U_{\text {in }}=0.01$ \\
\hline Optimal shape for $U_{\text {in }}=0.001$ (Fig. 22(b)) & $71.6 \%$ & $35.5 \%^{*}$ & $25.2 \%^{*}$ \\
Optimal shape for $U_{\text {in }}=0.005$ (Fig. 22(a)) & $65.1 \%$ & $36.3 \%$ & $22.6 \%$ \\
Optimal shape for $U_{\text {in }}=0.01$ (Fig. 18(b)) & $62.3 \%$ & $34.4 \%$ & $24.3 \%$ \\
\hline
\end{tabular}

*does not meet the energy constraint

Fig. 22(b) (i.e. the one resulting from the optimization procedure with $U_{\text {in }}=0.001 \mathrm{~m} . \mathrm{s}^{-1}$ as inlet boundary condition). The same conclusion is stated for $U_{\text {in }}=0.005 \mathrm{~m} . \mathrm{s}^{-1}$ (third column), and for $U_{\text {in }}=0.01 \mathrm{~m} . \mathrm{s}^{-1}$ (fourth column), the shape displayed on Fig. 22(b) has the highest conversion rate but does not satisfy the energy constraint. Consequently, the reactor meeting the set of constraints with the highest conversion rate for $U_{\text {in }}=0.01 \mathrm{~m} . \mathrm{s}^{-1}$ results from the shape optimization with $U_{\text {in }}=0.01 \mathrm{~m} \cdot \mathrm{s}^{-1}$.

\section{Conclusion}

A geometry optimization based on the adjoint system method is developed for shape optimization of fixed-bed reactors. The corresponding algorithm is implemented within OpenFOAM software in order to determine the optimal shape of the packing where a surface reaction or an homogeneous first order reaction takes place. In both cases, the objective is to minimize the average concentration of the reactant at the reactor outlet. The optimization problem is subjected to constraints involving Navier-Stokes and mass balance equations, an iso-volume and energy constraints, and to two manufacturing constraints. The optimized packing of the reactor allows a significant improvement of the conversion rate, i.e. $2.7 \%$ for the (HR) case and $4.7 \%$ for the (SR) case (under iso-energy constraint).

However, the developed optimization approach shows some weaknesses that need to be 
adressed. The first one concerns the width constraints treatment which is performed by post-treatment of the mesh displacement. It suffers from excessive strictness and does not allow the constraints violation during the optimization process as opposed to a constraint directly included in the Lagrangian functional. It might be possible to improve this issue by treating the width constraints using penalization functions (Allaire et al., 2016). The second weakness is related to the computational load of a single optimization and particularly in the surface reaction case. Indeed, the optimization process takes between 7 and 10 days to converge on a $3.7 \mathrm{GHz}$ Xeon Dell Computer 5810 (and 2-3 days for the homogeneous case). The most CPU time consuming step is the resolution of the PDEs by OpenFOAM. In our case, the computational time could not be decreased using parallel computing because the optimization method is iterative and the number of cells is between 50,000 and 120,000. However, it could be improved by modifying some numerical schemes (e.g. the use of SIMPLER algorithm instead of SIMPLE one) but the saved time would be marginal. Another way to reduce the CPU time required for CFD resolution could involve the use of surrogate models (Rabhi et al., 2018) which approximate the CFD solution needed by the shape optimization algorithm. The convergence of the iteration algorithm could also be accelerated using a line search method to determine the optimal step $t$ instead of using a fixed value for this parameter. This would limit the number of iterations required to achieve the convergence and consequently the number of PDEs resolutions. Finally, the use of a geometry optimization algorithm may not be appropriate since it preserves the topology of the reactor and the initial topology could not be the optimal topology. Therefore, it could be interesting to optimize the reactor using a topology optimization approach first and then refine the resulting shape using geometry optimization.

\section{Acknowledgment}

The authors are grateful to "Institut Carnot Énergie et Environnement de Lorraine (ICEEL)" for its financial support.

\section{Nomenclature}

\section{Latin symbols}

$\begin{array}{lll}C & \text { concentration field of the reactant } & \mathrm{mol} . \mathrm{m}^{-3} \\ \mathcal{C} & \text { set of constraints of the optimization problem } & - \\ C^{\prime} & \text { sensitivity of } C \text { with respect to the variation of } \Omega & \mathrm{mol} . \mathrm{m}^{-4} \\ C_{a} & \text { adjoint state of } C & \mathrm{~mol} \cdot \mathrm{m}^{-3} \\ C_{\mathcal{E}} & \text { energy dissipation constraint } & \mathrm{m}^{5} \cdot \mathrm{s}^{3} \\ C_{\mathrm{in}} & \text { reactant inlet concentration } & \mathrm{mol} \cdot \mathrm{m}^{-3} \\ C_{\mathcal{V}} & \text { iso-volume constraint } & \mathrm{m}^{3} \\ \mathcal{D} & \text { diffusion coefficient of the reactant in the solvent } & \mathrm{m}^{2} \cdot \mathrm{s}^{-1} \\ d_{\text {min }}^{\text {channel }} & \text { minimal width of channels } & \mathrm{m} \\ d_{\text {min }}^{\text {obstacle }} & \text { minimal thickness of obstacles } & \mathrm{m}\end{array}$




$\begin{array}{lll}G & \text { shape gradient } & - \\ J & \text { performance index } & - \\ k & \text { kinetic constant } & \mathrm{s}^{-1} \\ \mathcal{L} & \text { Lagrangian of the optimization problem } & - \\ \boldsymbol{n} & \text { boundary normal vector } & - \\ p & \text { kinematic pressure field } & \mathrm{Pa} \cdot \mathrm{m}^{3} \cdot \mathrm{kg}^{-1} \\ p^{\prime} & \text { sensitivity of } p \text { with respect to the variation of } \Omega & \mathrm{Pa} \cdot \mathrm{m}^{2} \cdot \mathrm{kg}^{-1} \\ p_{a} & \text { pressure of the adjoint state to }(\boldsymbol{U}, p) & \mathrm{Pa} \cdot \mathrm{m}^{3} \cdot \mathrm{kg}^{-1} \\ Q & \text { inlet flow rate } & \mathrm{m}^{3} \cdot \mathrm{s}^{-1} \\ \text { s.t. } & \text { subject to } & - \\ t & \text { step of the optimization method } & - \\ \boldsymbol{U} & \text { fluid flow velocity } & \mathrm{m} . \mathrm{s}^{-1} \\ \boldsymbol{U}^{\prime} & \text { sensitivity of } \boldsymbol{U} \text { with respect to the variation of } \Omega & \mathrm{s} \\ \boldsymbol{U}_{\boldsymbol{a}} & \text { velocity of the adjoint state to }(\boldsymbol{U}, p) & \mathrm{m} . \mathrm{s}^{-1} \\ \boldsymbol{U}_{\text {in }} & \text { fluid velocity profile imposed at the reactor inlet } & \mathrm{m} . \mathrm{s}^{-1} \\ \boldsymbol{V} & \text { vector field representing the displacement of the mesh } & \mathrm{m} \\ \mathcal{V}(\Omega) & \text { volume of } \Omega & \mathrm{m}^{2}\end{array}$

\section{Symboles grecs}

$\beta_{\mathcal{V}} \quad$ volume constraint update parameter

$\beta_{\mathcal{E}} \quad$ energy constraint update parameter

$\gamma$ parameter allowing to adjust the diffusion of the mesh when $\mathrm{m}^{2}$ moving it

$\Gamma \quad$ free boundary of the domain $\Omega$

$\Gamma_{\text {in }} \quad$ fluid inlet of $\Omega$

$\Gamma_{\text {out }} \quad$ fluid outlet of $\Omega$

$\Gamma_{\text {lat }} \quad$ lateral wall of $\Omega$

$\delta \quad$ distance between the boundary $\Gamma_{\text {out }}$ and the center of the ad- $\mathrm{m}$ jacent cell

$\partial \Omega \quad$ union of the boundaries of $\Omega$

$\lambda_{\mathcal{E}} \quad$ Lagrange multiplier associated to the energy constraint

$\lambda_{\mathcal{V}} \quad$ Lagrange multiplier associated to the volume constraint

$\nu \quad$ fluid kinematic viscosity

$\Omega \quad$ studied domain

\section{Indice}

$i \quad$ iteration of the shape optimization algorithm

a refer to an adjoint state

in refer to reactor inlet $\Omega$ 


\section{References}

G. Allaire, F. Jouve, and G. Michailidis, 2016. Thickness control in structural optimization via a level set method. Structural and Multidisciplinary Optimization, 53(6), 1349-1382, http://dx.doi.org/10.1007/s00158-016-1453-y.

G. Allaire, C. Dapogny, R. Estevez, A. Faure, and G. Michailidis, 2017. Structural optimization under overhang constraints imposed by additive manufacturing technologies. Journal of Computational Physics, 351, 295-328, http://dx.doi.org/10.1016/j.jcp.2017.09.041.

G. Allaire and M. Schoenauer, 2007. Conception optimale de structures, 58. SpringerVerlag, Berlin, ISBN : 978-3-540-36710-9, http://dx.doi.org/10.1007/978-3-540-36856-4.

D. Attali, 1995. Squelettes et graphes de Voronoï 2D et 3D. PhD thesis, Université Joseph Fourier - Grenoble I.

G. W. Burgreen, O. Baysal, and M. E. Eleshaky, 1994. Improving the efficiency of aerodynamic shape optimization. AIAA journal, 32(1), 69-76, http://dx.doi.org/10.2514/3.11952.

A. Courtais, 2021. Conceptions optimales de réacteurs à lit fixe par fabrication additive. $\mathrm{PhD}$ thesis, Université de Lorraine.

A. Courtais, F. Lesage, Y. Privat, P. Frey, and A. M. Latifi, 2019. Adjoint system method in shape optimization of some typical fluid flow patterns. Computer Aided Chemical Engineering, 46, 871-876. http://dx.doi.org/10.1016/B978-0-12-818634-3.50146-6.

A. Courtais, A. M. Latifi, F. Lesage, and Y. Privat, 2021. Shape optimization of fixed-bed reactors in process engineering. Accepted in SIAM Journal of Applied Mathematics.

C. Dapogny, P. Frey, F. Omnès, and Y. Privat, 2018. Geometrical shape optimization in fluid mechanics using FreeFem ++ . Structural and Multidisciplinary Optimization, 58 (6), 2761-2788, http://dx.doi.org/10.1007/s00158-018-2023-2.

X. D. De La Sablonière, B. Mauroy, and Y. Privat, 2011. Shape minimization of the dissipated energy in dyadic trees. Discrete and Continuous Dynamical Systems - Series B, 16(3), 767-799, http://dx.doi.org/10.3934/dcdsb.2011.16.767.

X. Dong and X. Liu, 2020. Multi-objective optimal design of microchannel cooling heat sink using topology optimization method. Numerical Heat Transfer; Part A: Applications, 77(1), 90-104, http://dx.doi.org/10.1080/10407782.2019.1682872.

F. Feppon, G. Allaire, and C. Dapogny, 2020. A variational formulation for computing shape derivatives of geometric constraints along rays. ESAIM: Mathematical Modelling and Numerical Analysis, 54(1), 181-228, http://dx.doi.org/10.1051/m2an/2019056. 
I. P. R. Grundtvig, A. E. Daugaard, J. M. Woodley, K. V. Gernaey, and U. Krühne, 2017. Shape optimization as a tool to design biocatalytic microreactors. Chemical Engineering Journal, 322, 215-223, http://dx.doi.org/10.1016/j.cej.2017.03.045.

J. Hadamard, 1908. Mémoire sur le problème d'analyse relatif à l'équilibre des plaques élastiques encastrées, 33. Imprimerie nationale.

A. Henrot and M. Pierre, 2005. Variation et optimisation de forme, 48. Springer-Verlag, Berlin, http://dx.doi.org/10.1007/3-540-37689-5.

A. Henrot and Y. Privat, 2010. What is the optimal shape of a pipe? Archive for Rational Mechanics and Analysis, 196(1), 281-302, http://dx.doi.org/10.1007/s00205-009-0243-8.

R. M. Hicks and P. A. Henne, 1978. Wing design by numerical optimization. Journal of Aircraft, 15(7), 407-412, http://dx.doi.org/10.2514/3.58379.

G. Holzinger, 2015. OpenFOAM: A little User-Manual. CD-Laboratory-Particulate Flow Modelling, Johannes Keplper University: Linz, Austria.

S. Hoseini, G. Najafi, B. Ghobadian, and A. Akbarzadeh, 2020. Impeller shapeoptimization of stirred-tank reactor: Cfd and fluid structure interaction analyses. Chemical Engineering Journal, 127497, http://dx.doi.org/10.1016/j.cej.2020.127497.

S. Kambampati, H. Chung, and H. A. Kim, 2021. A discrete adjoint based level set topology optimization method for stress constraints. Computer Methods in Applied Mechanics and Engineering, 377, 113563, http://dx.doi.org/10.1016/j.cma.2020.113563.

W. Karush, 2014. Minima of functions of several variables with inequalities as side conditions. Traces and Emergence of Nonlinear Programming, 217-245, http://dx.doi.org/10.1007/978-3-0348-0439-4_10.

H. W. Kuhn and A. W. Tucker, 2014. Nonlinear programming. Traces and Emergence of Nonlinear Programming, 247-258. Springer.

B. Kundu, 2007. Performance and optimum design analysis of longitudinal and pin fins with simultaneous heat and mass transfer: Unified and comparative investigations. Applied Thermal Engineering, 27(5-6), 976-987, http://dx.doi.org/10.1016/j.applthermaleng.2006.08.003.

O. Levenspiel, 1999. Chemical reaction engineering. John Wiley \& Sons, ISBN : 978-0471-25424-9.

R. Liang and Z. Yuan, 2020. Computational shape optimization of microreactors based on cfd simulation and surrogate model driven optimization. Computer Aided Chemical Engineering, 48, 925-930. http://dx.doi.org/10.1016/B978-0-12-823377-1.50155-5.

L. Lin, R. Wu, and X. X. Zhang, 2011. Optimization for geometric parameters of micro-channel heat sink using inverse problem method. Journal of Zhejiang University (Engineering Science), 45(4), 734-740, http://dx.doi.org/10.3785/j.issn.1008973X.2011.04.024. 
S. Ozguc, L. Pan, and J. A. Weibel, 2021. Topology optimization of microchannel heat sinks using a homogenization approach. International Journal of Heat and Mass Transfer, 169, 120896, http://dx.doi.org/10.1016/j.ijheatmasstransfer.2020.120896.

S. V. Patankar, 1980. Numerical heat transfer and fluid flow. Taylor \& Francis, ISBN : 978-1-31527-513-0.

A. Rabhi, A. Chkifa, S. Benjelloun, and A. Latifi, 2018. Surrogate-based modeling in flotation processes. Computer Aided Chemical Engineering, 43, 229-234. http://dx.doi.org/10.1016/B978-0-444-64235-6.50041-3.

J. Reuther, J. J. Alonso, M. J. Rimlinger, and A. Jameson, 1999. Aerodynamic shape optimization of supersonic aircraft configurations via an adjoint formulation on distributed memory parallel computers. Computers \& fluids, 28(4-5), 675-700, http://dx.doi.org/10.2514/6.1996-4045.

O. Tonomura, M. Kano, and S. Hasebe, 2010. Shape optimization of microchannels using CFD and adjoint method. Computer Aided Chemical Engineering, 28, 37-42. http://dx.doi.org/10.1016/S1570-7946(10)28007-0.

J. Villermaux, 1993. Génie de la réaction chimique. Technique et Documentation, ISBN : 978-2-85206-759-2.

H. G. Weller, G. Tabor, H. Jasak, and C. Fureby, 1998. A tensorial approach to computational continuum mechanics using object-oriented techniques. Computers in Physics, 12(6), 620, http://dx.doi.org/10.1063/1.168744.

M. Zhou, H. Lian, O. Sigmund, and N. Aage, 2018. Shape morphing and topology optimization of fluid channels by explicit boundary tracking. International Journal for Numerical Methods in Fluids, 88(6), 296-313, http://dx.doi.org/10.1002/fld.4667. 